\title{
Studies on Flavin Derivatives
}

\author{
The Crystal Structure of 5-Acetyl-9-bromo-1,3,7,8-tetramethyl-1,5- \\ dihydroalloxazine
}

\author{
MARIE LEIJONMARCK and PER-ERIK WERNER
}

\begin{abstract}
Institute of Inorganic and Physical Chemistry, University of Stockholm,
\end{abstract} S-104 05 Stockholm 50, Sweden

\begin{abstract}
The crystal structure of 5-acetyl-9-bromo-1,3,7,8-tetramethyl-1,5dihydroalloxazine, $\mathrm{C}_{16} \mathrm{H}_{17} \mathrm{BrN}_{4} \mathrm{O}_{3}$, has been determined and refined from three-dimensional $\mathrm{X}$-ray film data. The crystals are orthorhombic with space group $P n a 2_{1}$ and unit cell dimensions $a=24.771, b=7.500$, and $c=17.601 \AA$. The structure contains two molecules per asymmetric unit. A comparison between the present structure and the crystal structure of 5-acetyl-9-bromo-1,3,7,8,10-pentamethyl-1,5-dihydroisoalloxazine ${ }^{1}$ is made. A correction function for non-linearity in relative optical density vs. X-ray exposure curve has been tested in this structure determination.
\end{abstract}

$\mathrm{T}$ he structure determination of 5-acetyl-9-bromo-1,3,7,8-tetramethyl-1,5dihydroalloxazine, $\mathrm{C}_{16} \mathrm{H}_{17} \mathrm{BrN}_{4} \mathrm{O}_{3}$, was undertaken in order to test the reliability of $\mathrm{X}$-ray intensities calculated from irregularly-shaped diffraction spots by a modified version of a computer program system for a SAAB film scanner. ${ }^{2}$ In the original program system, published by one of us in a previous paper, ${ }^{3}$ it was assumed that a linear dependence existed between $\mathrm{X}$-ray exposure and measured optical density. It has been shown that by this program system, random errors in structure factors derived from precession photographs are usually less than $3 \%{ }^{4}$ Structure factors obtained from ordinary Weissenberg photographs have corresponding random errors less than $6 \%{ }^{4}$ It has been found, however, that systematic errors of the same magnitude as the random errors can be introduced if a completely linear dependence is assumed between $\mathrm{X}$-ray exposure and measured relative optical densities, $D^{\prime}$, in the range $0.07<D^{\prime}<1.4$. The relative optical density is then defined as ${ }^{10} \mathrm{log}$ (background transmission/spot transmission). This has been further discussed in a publication of the crystal structure of 5-diethyl3,7,8,10-tetramethyl-1,5-dihydroisoalloxazine trihydrate. ${ }^{5}$

The terms random error and systematic error are not used here in a very strict sense. This, because systematic errors in intensity measurements cause 
errors in film factors and thus contribute to random error in average intensities from different photographs.

The present structure determination was undertaken in order to test the influence of a correction function

$$
D_{\text {corr }}^{\prime}=D^{\prime}\left(1+k D^{\prime}\right)
$$

used in the integration of X-ray diffraction intensities from $D^{\prime}$-values obtained by a SAAB film scanner on-line to an IBM 1800 process controller. An equation of this kind for correction of optical density was first proposed by Mammi et al. ${ }^{6}$ For a number of crystals of different compounds, a value of $k=0.5$ has been found through calibration with wedges. This value has been used throughout the present structure determination.

A comparison between the present structure and the crystal structure of 5-acetyl-9-bromo-1,3,7,8,10-pentamethyl-1,5-dihydroisoalloxazine ${ }^{1}$ is also made.

\section{DATA COLLECTION}

Yellow single crystals of 5-acetyl-9-bromo-1,3,7,8-tetramethyl-1,5-dihydroalloxazine, $\mathrm{C}_{16} \mathrm{H}_{12} \mathrm{BrN}_{4} \mathrm{O}_{3}$, were prepared by Dr. L. Maròn at this Institute. Preliminary rotation and Weissenberg photographs showed systematic absences for $0 k l$ with $k+l$ odd, and $h 0 l$ with $h$ odd. This is characteristic of the two space groups Pnam and Pna2 $2_{1}$ Lattice parameters of the orthorhombic unit cell were calculated from an X-ray powder diffraction photograph taken with monochromatized $C u K \alpha_{1}$ radiation in a Guinier-Hägg focussing camera with potassium chloride $(a=6.2930 \AA)$ as an internal standard. The parameters obtained are given in Table 1.

Table 1. Crystal data.

Lattice constants "
Cell volume
Density (X-ray)
Analysis
Molecules/unit cell
Absent reflections
Space group
General position

$$
\begin{aligned}
& a=24.771(7) \AA \\
& b=7.500(3) \AA \\
& c=17.601(5) \AA \\
& V=3270.0 \AA^{3} \\
& d=1.60 \mathrm{~g} / \mathrm{cm}^{3} \\
& \mathrm{C} 49.0 ; \mathrm{H} 4.5 ; \mathrm{N} 14.2 ; \mathrm{Br} 20.4 \text {. Calc. for } \mathrm{C}_{16} \mathrm{H}_{17} \mathrm{BrN}_{4} \mathrm{O}_{3}: \\
& \mathrm{C} 48.9 ; \mathrm{H} 4.3 ; \mathrm{N} 14.2 ; \mathrm{Br} 20.3 ; \mathrm{O} 12.2 \\
& Z=8 \\
& 0 k l \text { with } k+l=2 n+1, \text { and } h k 0 \text { with } h=2 n+1 \\
& \text { Pna } 21 \\
& \left(x y z ; \bar{x}, \bar{y}, \frac{1}{2}+z ; \frac{1}{2}-x, \frac{1}{2}+y, \frac{1}{2}+z ; \frac{1}{2}+x, \frac{1}{2}-y, z\right)
\end{aligned}
$$

A somewhat irregularly-shaped rectangular plate, $0.08 \mathrm{~mm}(a) \times 0.40 \mathrm{~mm}(b) \times 0.31 \mathrm{~mm}$ (c) was mounted and rotated around the c-axis. Non-integrated Weissenberg photographs were taken by the multiple film technique with $\mathrm{Cu} K \alpha$ radiation. Relative intensities of the 15 layer lines, $h k 0-h k 14$, were measured with a SAAB film scanner ${ }^{8}$ on-line to an IBM 1800 process controller.

Small splittings in the diffraction spots on the first three layers were observed. Since the spot integration technique used in the computer program system ${ }^{3}$ is applicable to arbitrary spot shapes, this should not be considered deleterious. Integrated intensities for all spots with a relative optical density $D^{\prime}={ }^{10} \log$ (background transmission/spot transmission) in the range $0.07<D^{\prime}<1.2$ were calculated. In the integration procedure, all $D^{\prime}$-values were corrected by the equation $D_{\text {cerr }}^{\prime}=D^{\prime}\left(1+0.5 D^{\prime}\right.$ ) (see above). The cross section of the light beam used was $60 \mu \mathrm{m} \times 90 \mu \mathrm{m}$, and about $80 \%$ of the integrated spots contained $60-130$ individual density readings (i.e. spot size $=0.3-0.7 \mathrm{~mm}^{2}$ ). 
The film factors were calculated by the method of Monahan et al. ${ }^{9}$ As might be expected, the most extreme film factor values were found for the layers $h k 13$ and $h k 14$. They were all above 3.2. For all 40 film factors calculated, the average $2.86 \pm 0.04$ was obtained. This may be compared with the value 2.93 given by Morimoto and Uyeda ${ }^{10}$ for the film type used, Ilford Industrial G. The validity of the correction function applied to the relative optical densities is also confirmed by the zero slopes in plots of film factors versus intensity. Such tests were performed by least-squares calculations of the slopes. The calculated slopes had different signs, and their absolute values were less than three standard deviations of the slopes. Because of the small standard deviations, this should be considered as a very sensitive demonstration of the absence of systematic errors in the intensity calculations.

The reflections were corrected for absorption $\left(\mu=40.1 \mathrm{~cm}^{-1}\right)$ and Lorentz-polarization effects. The total number of independent reflections was then 1556.

\section{STRUCTURE DETERMINATION AND REFINEMENT}

A three-dimensional Patterson synthesis, $P(u v w)$, was calculated. In order to determine the correct space group, the Harker sections for the two possible space groups Pnam and Pna2, were investigated. Because of the absence of any high peaks along $(0,0, w)$, except the origin peak, only two fourfold bromine positions with $z=0.5$ are possible, if Pnam is assumed. From this follows that bromine-bromine peaks should occur only at $w=0.5$. This was not confirmed, however, since high peaks were found also at other positions. Therefore, Pnam was excluded.

The bromine positions were thus derived for space group $P n a 2_{1}$ from the prominent peaks in $P(u v w)$ and refined by least-squares treatment. The $R$ index $\left[R=\sum|| k F_{o}|-| F_{\mathrm{c}}|| / \sum\left|k F_{\mathrm{o}}\right|\right]$ for the bromine atoms alone was 0.33 . The search for other atoms in the structure was pursued by Fourier techniques. By successive $F_{0}$-syntheses, all the non-hydrogen atoms were found.

Initial least-squares refinement was carried out with a block-diagonal matrix program (SFLS, written by S. Åsbrink at this Institute, and by C.-I. Brändén, University of Uppsala, and modified by B. G. Brandt at this Institute). A full-matrix program (LALS, originally written by Gantzel, Sparks and Trueblood, ${ }^{11}$ and modified by R. Liminga, J.-O. Lundgren and C.-I. Brändén, University of Uppsala, and by B. G. Brandt and A. G. Nord at this Institute) was used in later stages. The scattering factor curves used for oxygen, nitrogen, and carbon were those of Freeman, ${ }^{12}$ and for bromine that of Cromer and Waber, ${ }^{13}$ corrected for the real part of the anomalous dispersion coefficient. The weights were calculated according to the expression suggested by Cruickshank ${ }^{14}$

$$
w=\left(a+F_{\mathrm{o}}+c F_{\mathrm{o}}^{2}\right)^{-1}
$$

with the empirically chosen values $a=3.0$ and $c=0.04$. The parameters refined were scale factors, atomic coordinates, anisotropic temperature factors for bromine, and isotropic temperature factors for the remaining non-hydrogen atoms. The refinement was terminated when all shifts in the parameters were less than a quarter of their corresponding standard deviations. The $R$-value obtained was then 0.069 . A refinement of the same parameters without the

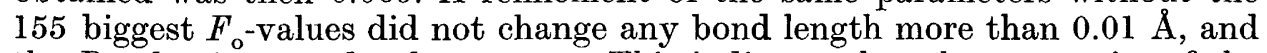
the $R$-value increased only to 0.071 . This indicates that the accuracies of the

Acta Chem. Scand. 25 (1971) No. 6 
two groups of structure factors are comparable, and that no appreciable extinction occurs.

Hydrogen atom positions were derived partly from a difference synthesis, and partly from ordinary stereochemical considerations. The $R$-value, calculated with the hydrogen atoms included with an over all temperature factor of $4 \AA^{2}$, was 0.063 . The hydrogen parameters were not refined, but they were included in the structure factor calculations in later anisotropic refinement of the structure.

It is well known that because of the strong correlations between anisotropic temperature factors and scale factors, it is usually not possible to make an adequate anisotropic refinement of a structure, unless diffraction data are collected from different crystal settings, allowing the structure factors to be put on a common scale. Furthermore, because of the occurrence of two molecules in the asymmetric unit in the present case, the ratio of refinable parameters to observed structure factors might be too big. On the other hand, the presence of two almost equivalent molecules in the asymmetric unit makes it possible to judge the physical relevance of further refinements. Therefore, two least-squares cycles with constant scale factors were performed with anisotropic temperature factors for the bromine atoms and for all carbon, nitrogen, and oxygen atoms in one of the two molecules in the asymmetric unit, while the other molecule was isotropically refined. Thereafter, the other molecule was refined with anisotropic temperature factors, while the remaining parameters were kept constant. The refinement was continued until no shift

Table 2. Weight analysis obtained in the final cycle of the least-squares refinement of 5-acetyl-9-bromo-1,3,7,8-tetramethyl-1,5-dihydroalloxazine.

The averages $\overline{w\left(\left|F_{\mathrm{o}}\right|-\left|F_{\mathrm{c}}\right|\right)^{2}}=\overline{w \Delta^{2}}$ are normalized.

\begin{tabular}{cccccc}
\hline $\begin{array}{c}\text { Interval } \\
\text { sin } \theta\end{array}$ & $\begin{array}{c}\text { Number of } \\
\text { independent } \\
\text { reflections }\end{array}$ & $\overline{w \Delta^{2}}$ & $\begin{array}{c}\text { Interval } \\
F_{\text {obs }}\end{array}$ & $\begin{array}{c}\text { Number of } \\
\text { independent } \\
\text { reflections }\end{array}$ & $\overline{w \Delta^{2}}$ \\
\hline $0.0-0.441$ & 269 & 1.55 & $0.0-12.8$ & 154 & 1.17 \\
$0.441-0.556$ & 286 & 1.01 & $12.8-16.1$ & 156 & 1.06 \\
$0.556-0.636$ & 253 & 0.78 & $16.1-19.7$ & 155 & 0.82 \\
$0.636-0.700$ & 205 & 0.81 & $19.7-23.1$ & 156 & 0.85 \\
$0.700-0.754$ & 167 & 0.87 & $23.1-27.5$ & 156 & 0.61 \\
$0.754-0.801$ & 112 & 0.81 & $27.5-31.5$ & 155 & 0.77 \\
$0.801-0.843$ & 88 & 0.71 & $31.5-37.3$ & 156 & 0.96 \\
$0.843-0.882$ & 76 & 0.81 & $37.3-45.1$ & 155 & 1.00 \\
$0.882-0.917$ & 52 & 1.26 & $45.1-60.5$ & 156 & 0.98 \\
$0.917-0.950$ & 42 & 1.45 & $60.5-138.8$ & 156 & 1.78 \\
\hline
\end{tabular}

was greater than $10 \%$ of the corresponding standard deviation. The final $R$-value was 0.046 . The weight analysis obtained in the final cycle is given in Table 2. The positional and thermal parameters obtained for the nonhydrogen atoms are given in Tables 3 and 4 . Observed and calculated structure factors are listed in Table 5. The differences between the corresponding bond 
Table 3. Atomic parameters with their standard deviations.

\begin{tabular}{|c|c|c|c|c|c|c|}
\hline Atom & \multicolumn{2}{|l|}{$x / a$} & \multicolumn{2}{|l|}{$y / b$} & \multicolumn{2}{|l|}{$z / c$} \\
\hline $\mathrm{Br}$ & 0.58761 & (5) & 0.43480 & (17) & 0.25 & \\
\hline$N(1)$ & 0.4124 & (4) & 0.6876 & (13) & 0.3458 & (5) \\
\hline$C(2)$ & 0.3712 & (5) & 0.7836 & (16) & 0.3796 & (9) \\
\hline $\mathbf{N}(3)$ & 0.3710 & (3) & 0.7960 & (12) & 0.4559 & (7) \\
\hline $\mathrm{C}(4)$ & 0.4091 & (4) & 0.7126 & (15) & 0.5038 & (9) \\
\hline$C(4 a)$ & 0.4470 & (4) & 0.6004 & (14) & 0.4659 & (7) \\
\hline$N(5)$ & 0.4845 & (3) & 0.4994 & (10) & 0.5068 & (5) \\
\hline $\mathrm{C}(5 \mathrm{a})$ & 0.5374 & (3) & 0.4963 & (12) & 0.4709 & (7) \\
\hline$C(6)$ & 0.5855 & (4) & 0.4878 & (14) & 0.5147 & (7) \\
\hline $\mathrm{C}(7)$ & 0.6337 & (4) & 0.4535 & (12) & 0.4792 & (8) \\
\hline $\mathrm{C}(8)$ & 0.6369 & (4) & 0.4376 & (11) & 0.4001 & (7) \\
\hline $\mathrm{C}(9)$ & 0.5895 & (4) & 0.4628 & (13) & 0.3589 & (7) \\
\hline$C(9 a)$ & 0.5392 & (4) & 0.4910 & (11) & 0.3945 & (7) \\
\hline $\mathrm{N}(10)$ & 0.4918 & (3) & 0.5202 & (10) & 0.3525 & (5) \\
\hline $\mathrm{C}(10 \mathrm{a})$ & 0.4504 & (4) & 0.5992 & (12) & 0.3891 & (7) \\
\hline $\mathrm{C}(11)$ & 0.4190 & (6) & 0.7023 & (17) & 0.2593 & (9) \\
\hline $\mathrm{O}(12)$ & 0.3354 & (4) & 0.8558 & (12) & 0.3396 & (6) \\
\hline $\mathrm{C}(13)$ & 0.3284 & (5) & 0.8995 & (18) & 0.4938 & (9) \\
\hline $\mathrm{O}(14)$ & 0.4083 & (3) & 0.7338 & (14) & 0.5726 & (7) \\
\hline $\mathrm{C}(15)$ & 0.4709 & (4) & 0.3598 & (16) & 0.5540 & (7) \\
\hline $\mathrm{C}(15 \mathrm{a})$ & 0.4114 & (4) & 0.3179 & (16) & 0.5609 & (8) \\
\hline $\mathrm{O}(15 \mathrm{~b})$ & 0.5054 & (3) & 0.2686 & (11) & 0.5856 & (5) \\
\hline $\mathrm{C}(17)$ & 0.6836 & (4) & 0.4312 & $(15)$ & 0.5287 & (8) \\
\hline $\mathrm{C}(18)$ & 0.6883 & (5) & 0.3998 & (17) & 0.3581 & (8) \\
\hline
\end{tabular}

Mol. II.

\begin{tabular}{lllllll}
\hline & & & & & & \\
$\mathrm{Br}$ & 0.01565 & $(5)$ & 0.47532 & $(14)$ & 0.40442 & $(11)$ \\
$\mathrm{N}(1)$ & 0.0868 & $(3)$ & 0.6335 & $(12)$ & 0.6590 & $(6)$ \\
$\mathrm{C}(2)$ & 0.1075 & $(4)$ & 0.7135 & $(14)$ & 0.7217 & $(7)$ \\
$\mathrm{N}(3)$ & 0.1619 & $(3)$ & 0.7506 & $(11)$ & 0.7218 & $(5)$ \\
$\mathrm{C}(4)$ & 0.1964 & $(4)$ & 0.7232 & $(13)$ & 0.6583 & $(6)$ \\
$\mathrm{C}(4 \mathrm{a})$ & 0.1734 & $(4)$ & 0.6207 & $(13)$ & 0.6007 & $(6)$ \\
$\mathrm{N}(5)$ & 0.2033 & $(3)$ & 0.5699 & $(11)$ & 0.5348 & $(5)$ \\
$\mathrm{C}(5 \mathrm{a})$ & 0.1757 & $(4)$ & 0.5767 & $(13)$ & 0.4633 & $(6)$ \\
$\mathrm{C}(6)$ & 0.1980 & $(4)$ & 0.6177 & $(13)$ & 0.3957 & $(7)$ \\
$\mathrm{C}(7)$ & 0.1681 & $(6)$ & 0.6114 & $(14)$ & 0.3288 & $(7)$ \\
$\mathrm{C}(8)$ & 0.1151 & $(6)$ & 0.5641 & $(15)$ & 0.3288 & $(7)$ \\
$\mathrm{C}(9)$ & 0.0910 & $(4)$ & 0.5306 & $(13)$ & 0.3990 & $(9)$ \\
$\mathrm{C}(9 \mathrm{a})$ & 0.1201 & $(4)$ & 0.5359 & $(14)$ & 0.4674 & $(6)$ \\
$\mathrm{N}(10)$ & 0.0955 & $(3)$ & 0.5045 & $(11)$ & 0.5371 & $(6)$ \\
$\mathrm{C}(10 \mathrm{a})$ & 0.1188 & $(4)$ & 0.5822 & $(12)$ & 0.6000 & $(6)$ \\
$\mathrm{C}(11)$ & 0.0280 & $(4)$ & 0.6287 & $(18)$ & 0.6491 & $(8)$ \\
$\mathrm{O}(12)$ & 0.0789 & $(3)$ & 0.7520 & $(13)$ & 0.7779 & $(5)$ \\
$\mathrm{C}(13)$ & 0.1861 & $(5)$ & 0.8260 & $(20)$ & 0.7895 & $(7)$ \\
$\mathrm{O}(14)$ & 0.2423 & $(3)$ & 0.7816 & $(11)$ & 0.6602 & $(5)$ \\
$\mathrm{C}(15)$ & 0.2519 & $(4)$ & 0.4779 & $(14)$ & 0.5392 & $(6)$ \\
$\mathrm{C}(15 \mathrm{a})$ & 0.2697 & $(5)$ & 0.4170 & $(17)$ & 0.6143 & $(8)$ \\
$\mathrm{O}(15 \mathrm{~b})$ & 0.2774 & $(3)$ & 0.4409 & $(11)$ & 0.4812 & $(5)$ \\
$\mathrm{C}(17)$ & 0.1990 & $(6)$ & 0.6559 & $(19)$ & 0.2533 & $(9)$ \\
$\mathrm{C}(18)$ & 0.0822 & $(6)$ & 0.5567 & $(16)$ & 0.2552 & $(11)$ \\
\hline
\end{tabular}

Acta Chem. Scand. 25 (1971) No. 6 
Table 4. Thermal parameters. The $\beta$ values refer to the temperature factor $\exp \left[-\left(h^{2} \beta_{11}+k^{2} \beta_{22}+l^{2} \beta_{33}+h k \beta_{12}+h l \beta_{13}+k l \beta_{23}\right) \times 10^{-5}\right]$

$\mathrm{R}(\mathrm{i}), \mathrm{i}=1-3$, are the r.m.s. components of thermal displacement (in $\AA$ ) along the principal axes of the ellipsoid of thermal vibration (calculated by the program ORTEP ${ }^{15}$ ).

\begin{tabular}{lrrrrrrrrr}
\hline $\begin{array}{l}\text { Atom } \\
\text { Mol. I }\end{array}$ & $\beta_{11}$ & $\beta_{22}$ & $\beta_{33}$ & $\beta_{12}$ & $\beta_{13}$ & $\beta_{23}$ & $R(1)$ & $R(2)$ & $R(3)$ \\
\hline Br & & & & & & & & & \\
N(1) & 263 & 2319 & 347 & -109 & 193 & -45 & 0.207 & 0.255 & 0.307 \\
C(2) & 179 & 2338 & 310 & 110 & -126 & 2 & 0.193 & 0.250 & 0.266 \\
N(3) & 188 & 2180 & 515 & -61 & -176 & -261 & 0.211 & 0.252 & 0.305 \\
C(4) & 120 & 2143 & 544 & 196 & -75 & -322 & 0.183 & 0.241 & 0.303 \\
C(4a) & 116 & 2320 & 391 & -142 & -3 & -409 & 0.185 & 0.226 & 0.280 \\
N(5) & 92 & 1933 & 434 & 122 & -55 & 5 & 0.162 & 0.237 & 0.263 \\
C(5a) & 132 & 1543 & 334 & 7 & 36 & 163 & 0.198 & 0.205 & 0.237 \\
C(6) & 87 & 1492 & 400 & 140 & 27 & 288 & 0.157 & 0.201 & 0.259 \\
C(7) & 175 & 1701 & 384 & 64 & -118 & 50 & 0.205 & 0.224 & 0.266 \\
C(8) & 102 & 1325 & 564 & -84 & -55 & -438 & 0.164 & 0.195 & 0.305 \\
C(9) & 151 & 1287 & 458 & -5 & 95 & -42 & 0.191 & 0.208 & 0.275 \\
C(9a) & 165 & 1672 & 280 & -145 & 161 & -298 & 0.169 & 0.207 & 0.267 \\
N(10) & 140 & 1641 & 204 & -69 & -52 & 199 & 0.168 & 0.204 & 0.229 \\
C(10a) & 171 & 1461 & 234 & 149 & -126 & -83 & 0.169 & 0.198 & 0.252 \\
C(11) & 164 & 1424 & 254 & -91 & -72 & -87 & 0.181 & 0.207 & 0.235 \\
O(12) & 288 & 2595 & 492 & -184 & -147 & 371 & 0.249 & 0.268 & 0.327 \\
(13) & 242 & 2643 & 868 & 422 & -402 & 568 & 0.186 & 0.303 & 0.400 \\
O(14) & 147 & 2833 & 739 & 517 & 142 & -313 & 0.170 & 0.305 & 0.347 \\
C(15) & 160 & 4276 & 524 & 275 & 26 & -580 & 0.215 & 0.276 & 0.362 \\
C(15a) & 183 & 2248 & 264 & -136 & 135 & 14 & 0.179 & 0.245 & 0.265 \\
O(15b) & 167 & $\mathbf{2 4 7 9}$ & $\mathbf{4 5 7}$ & -181 & 131 & 228 & 0.200 & 0.271 & 0.283 \\
C(17) & 209 & 2850 & 457 & 269 & 8 & $\mathbf{7 0 1}$ & 0.221 & 0.256 & 0.321 \\
C(18) & 169 & 2017 & 538 & 183 & -140 & -367 & 0.212 & 0.231 & 0.310 \\
& 168 & 2374 & 637 & 122 & 179 & -327 & 0.204 & 0.263 & 0.330
\end{tabular}

Mol. II

\begin{tabular}{|c|c|c|c|c|c|c|c|c|c|}
\hline $\mathrm{Br}$ & 198 & 1806 & 619 & 85 & -383 & -12 & 0.183 & 0.227 & 0.354 \\
\hline $\mathbf{N}(1)$ & 126 & 1951 & 412 & -27 & -13 & 115 & 0.197 & 0.233 & 0.257 \\
\hline$C(2)$ & 179 & 1759 & 384 & 75 & 81 & 101 & 0.219 & 0.222 & 0.261 \\
\hline $\mathbf{N}(3)$ & 174 & 1953 & 211 & 90 & 15 & -132 & 0.179 & 0.227 & 0.244 \\
\hline$C(4)$ & 144 & 1972 & 104 & -50 & 9 & 141 & 0.126 & 0.211 & 0.239 \\
\hline$C(4 a)$ & 118 & 1623 & 206 & -20 & -68 & -127 & 0.161 & 0.204 & 0.218 \\
\hline $\mathbf{N}(\mathbf{5})$ & 141 & 1825 & 220 & 233 & 20 & -51 & 0.180 & 0.195 & 0.244 \\
\hline$C(5 a)$ & 145 & 1296 & 251 & 182 & -23 & 67 & 0.174 & 0.201 & 0.225 \\
\hline $\mathrm{C}(6)$ & 224 & 1589 & 177 & 150 & 63 & 23 & 0.163 & 0.208 & 0.269 \\
\hline$C(7)$ & 290 & 1300 & 250 & 156 & -55 & -13 & 0.190 & 0.196 & 0.303 \\
\hline $\mathrm{C}(8)$ & 277 & 1409 & 254 & 150 & -132 & -78 & 0.189 & 0.198 & 0.302 \\
\hline $\mathrm{C}(9)$ & 197 & 1216 & 487 & 79 & -250 & -115 & 0.185 & 0.201 & 0.313 \\
\hline$C(9 a)$ & 151 & 1707 & 176 & -18 & 2 & 214 & 0.159 & 0.217 & 0.226 \\
\hline $\mathrm{N}(10)$ & 123 & 1723 & 342 & -90 & -87 & 41 & 0.182 & 0.221 & 0.243 \\
\hline$C(10 a)$ & 130 & 1443 & 235 & 85 & 3 & -8 & 0.191 & 0.193 & 0.212 \\
\hline$C(11)$ & 159 & 2859 & 457 & 32 & 152 & -477 & 0.196 & 0.263 & 0.308 \\
\hline$O(12)$ & 212 & 3294 & 516 & -105 & 256 & -883 & 0.199 & 0.274 & 0.355 \\
\hline $\mathrm{C}(13)$ & 231 & 3488 & 262 & 14 & -66 & -612 & 0.183 & 0.269 & 0.326 \\
\hline$O(14)$ & 146 & 3191 & 356 & -199 & -64 & -483 & 0.192 & 0.239 & 0.313 \\
\hline$C(15)$ & 134 & 1955 & 247 & 79 & -74 & 228 & 0.171 & 0.219 & 0.242 \\
\hline$C(15 a)$ & 198 & 2772 & 415 & 445 & 29 & 288 & 0.217 & 0.250 & 0.310 \\
\hline$O(15 b)$ & 160 & 2913 & 365 & 348 & 120 & 137 & 0.192 & 0.246 & 0.304 \\
\hline$C(17)$ & 418 & 3110 & 307 & 360 & 170 & 319 & 0.207 & 0.288 & 0.375 \\
\hline$C(18)$ & 385 & 2182 & 450 & 344 & -385 & -274 & 0.214 & 0.241 & 0.385 \\
\hline
\end{tabular}


Table 5. Observed and calculated structure factors.
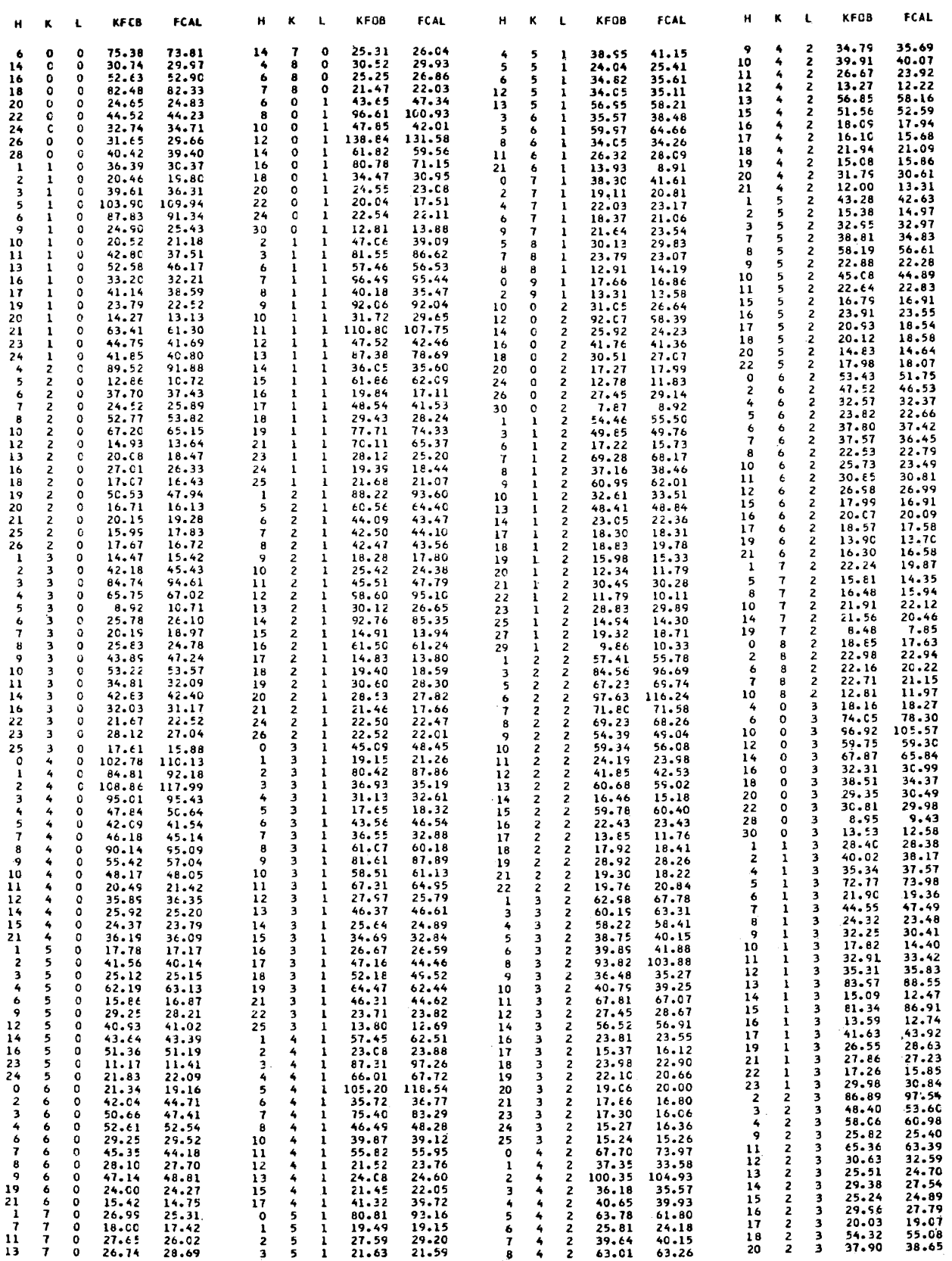
Table 5. Continued.

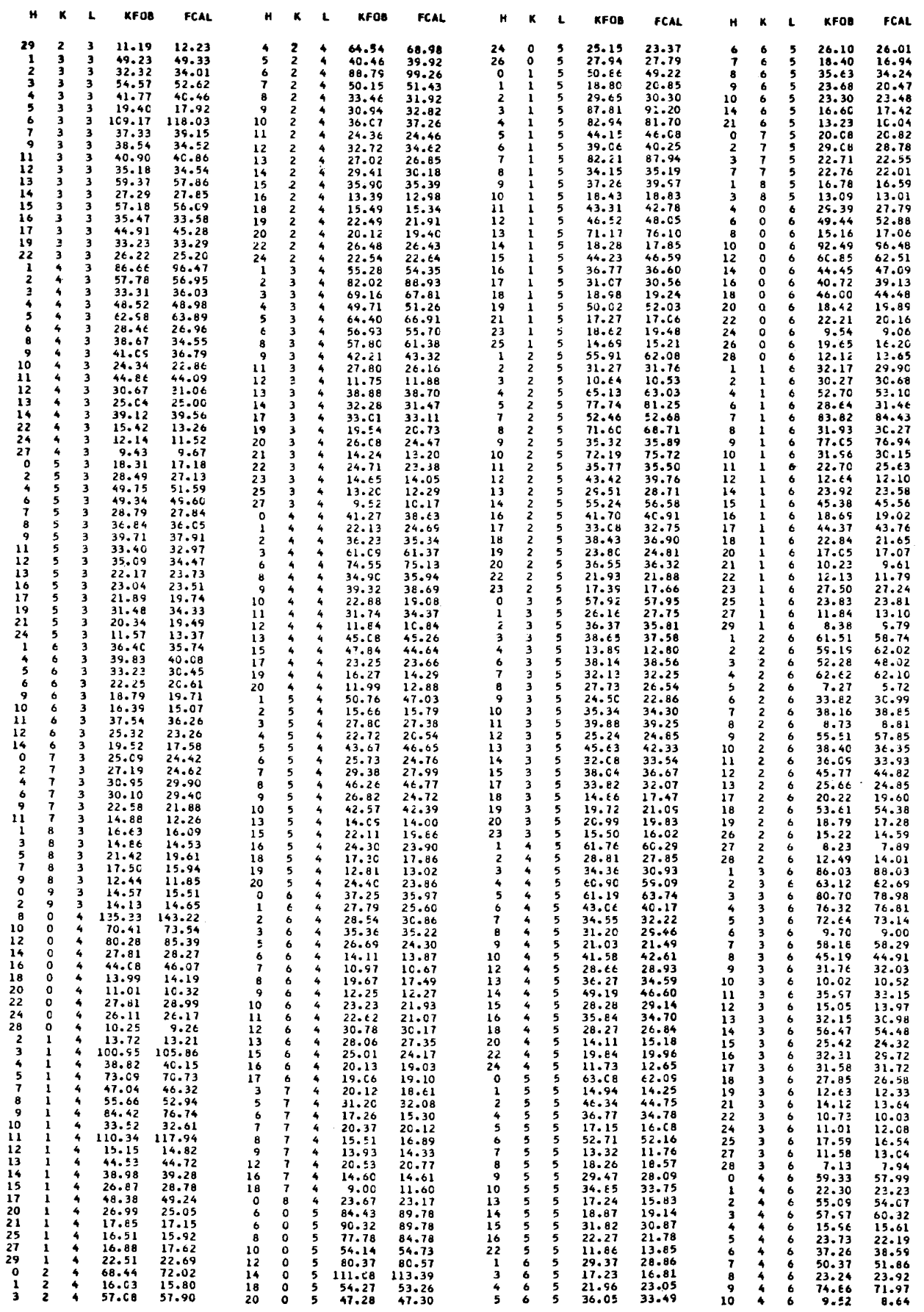


Table 5. Continued.

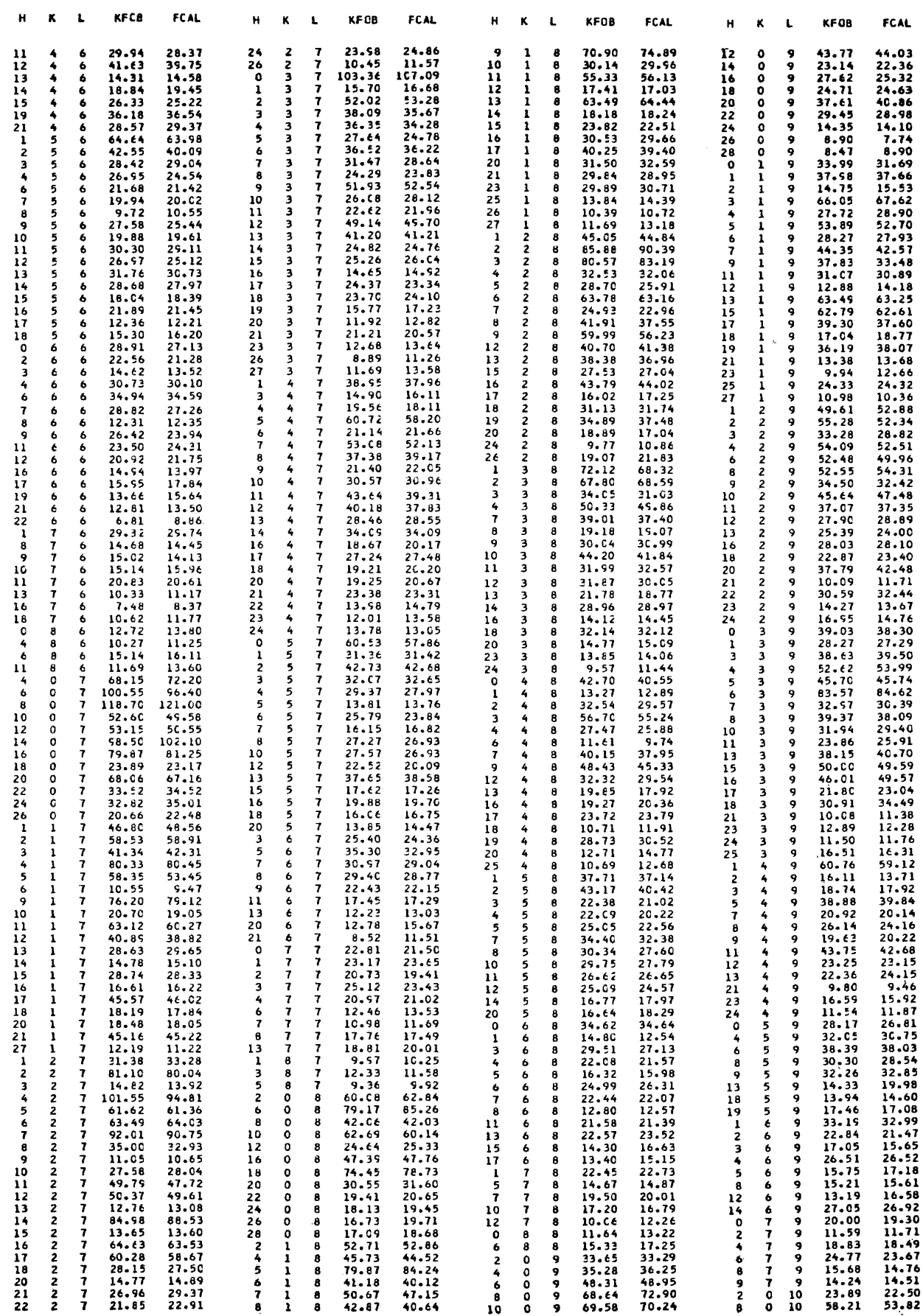


Table 5. Continued.

\begin{tabular}{|c|c|c|c|c|c|c|c|c|c|c|c|c|c|}
\hline H & $\begin{array}{ll}K & L\end{array}$ & $\mathrm{KFCB}$ & FCAL & $\mathrm{H}$ & k & I & KFCB & FCAL & H & L & $\mathrm{KFCB}$ & FCAL & H \\
\hline & $0 \quad 10$ & & 29.24 & & & & & & & & & & \\
\hline 12 & 0 io & $0 \quad 62.40$ & 62.55 & 9 & 2 & 11 & 17.81 & 16.54 & 11 & 212 & 11.57 & 12.61 & $\begin{array}{l}13 \\
14\end{array}$ \\
\hline 16 & $\therefore \quad 10$ & $0 \quad 19.03$ & 28.13 & 10 & & ii & 54.25 & 53.33 & 12 & 212 & 35.78 & 31.88 & 15 \\
\hline 16 & $0 \quad 10$ & $0 \quad 17.75$ & 19.78 & i & 3 & 11 & $\begin{array}{l}29.84 \\
13.01\end{array}$ & $\begin{array}{r}28.00 \\
12.84\end{array}$ & $\begin{array}{l}14 \\
15\end{array}$ & $\begin{array}{l}2 \\
2\end{array}$ & 25.70 & 25.25 & 3 \\
\hline $\begin{array}{l}18 \\
20 \\
20\end{array}$ & $\begin{array}{lll}0 & 10 \\
0 & 10\end{array}$ & $\begin{array}{l}0 \\
01.27 \\
0\end{array}$ & $\begin{array}{l}41.84 \\
21.35\end{array}$ & $\frac{1}{2}$ & $\begin{array}{l}3 \\
3\end{array}$ & $\begin{array}{l}11 \\
11\end{array}$ & $\begin{array}{l}\begin{array}{l}13.01 \\
36.76\end{array} \\
36\end{array}$ & $\begin{array}{l}12.84 \\
38.72\end{array}$ & $\begin{array}{l}15 \\
18\end{array}$ & $\begin{array}{ll}2 & 12 \\
2 & 12\end{array}$ & $\begin{array}{l}20.31 \\
10.56\end{array}$ & $\begin{array}{l}21.71 \\
11.43\end{array}$ & ${ }_{4}^{2}$ \\
\hline 24 & 010 & 27.26 & 27.32 & 3 & & ii & $\begin{array}{l}18.4 \epsilon \\
38.52\end{array}$ & $\begin{array}{l}19.10 \\
36.19\end{array}$ & $\begin{array}{l}19 \\
20\end{array}$ & $\begin{array}{ll}2 & 12 \\
2 & 12\end{array}$ & $\begin{array}{l}18.68 \\
25.05\end{array}$ & $\begin{array}{l}29.09 \\
24.06\end{array}$ & 6 \\
\hline 26 & $\begin{array}{ll}0 & 10 \\
1 & 10\end{array}$ & $\begin{array}{l}10.68 \\
0 \\
0\end{array}$ & $\begin{array}{l}11.74 \\
34.25\end{array}$ & 5 & $\begin{array}{l}3 \\
3\end{array}$ & $\begin{array}{l}11 \\
11\end{array}$ & $\begin{array}{l}38.52 \\
19.42\end{array}$ & $\begin{array}{l}36.19 \\
15.8 t\end{array}$ & $\begin{array}{l}20 \\
21\end{array}$ & $\begin{array}{ll}2 & 12 \\
2 & 12\end{array}$ & 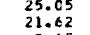 & $\begin{array}{l}244.86 \\
19.58\end{array}$ & $\begin{array}{r}8 \\
10\end{array}$ \\
\hline 3 & 110 & $0 \quad 48.57$ & $\begin{array}{r}48.54 \\
46.55\end{array}$ & 6 & & ii. & $\begin{array}{l}40.42 \\
27\end{array}$ & $\begin{array}{r}42.70 \\
229.51\end{array}$ & 22 & $\begin{array}{l}212 \\
3\end{array}$ & $\begin{array}{r}9.65 \\
19.21\end{array}$ & 8.16 & 12 \\
\hline & $\begin{array}{l}110 \\
110\end{array}$ & $\begin{array}{r}6.56 \\
0 \quad 24\end{array}$ & $\begin{array}{r}0.55 \\
21.22\end{array}$ & $\begin{array}{l}7 \\
8\end{array}$ & 3 & ${ }_{11}^{11}$ & $\begin{array}{l}27.17 \\
32.70\end{array}$ & $\begin{array}{r}29.51 \\
29.42\end{array}$ & $\frac{1}{2}$ & $\begin{array}{ll}3 & 12 \\
3 & 12\end{array}$ & $\begin{array}{l}19.21 \\
20.89\end{array}$ & $\begin{array}{l}19.30 \\
20.45\end{array}$ & 14 \\
\hline & $\begin{array}{ll}1 & 10 \\
1 & 10\end{array}$ & $\begin{array}{l}24.44 \\
30.54\end{array}$ & $\begin{array}{l}21.22 \\
35.21\end{array}$ & $\stackrel{8}{9}$ & ${ }_{3}^{3}$ & $\begin{array}{l}11 \\
11\end{array}$ & $\begin{array}{l}32.70 \\
22.24\end{array}$ & 20.10 & $\frac{2}{3}$ & $\begin{array}{ll}\begin{array}{l}3 \\
3\end{array} & 12 \\
3 & 12\end{array}$ & $\begin{array}{l}20.89 \\
32.52\end{array}$ & $\begin{array}{l}20.45 \\
34.91\end{array}$ & $\begin{array}{l}16 \\
18\end{array}$ \\
\hline & 10 & $\begin{array}{l}30.24 \\
0\end{array}$ & 10.91 & 10 & 3 & 11 & 22.57 & 21.46 & 5 & 312 & 16.7 .5 & 15.56 & 20 \\
\hline & 10 & 32.57 & 31.43 & 11 & 3 & ii & 29.59 & 29.19 & 7 & $\begin{array}{ll}3 & 12\end{array}$ & $3 c .83$ & 31.53 & 1 \\
\hline & 110 & $84.8 \mathrm{C}$ & 42.17 & 12 & 3 & 11 & 40.25 & $\begin{array}{r}38.50 \\
30.77\end{array}$ & 8 & $\begin{array}{ll}312 \\
312\end{array}$ & 31.30 & 31.14 & 3 \\
\hline & 110 & $\begin{array}{l}33.54 \\
60.74\end{array}$ & $\begin{array}{l}34.74 \\
67.07\end{array}$ & $\begin{array}{l}13 \\
14\end{array}$ & 3 & 11 & $\begin{array}{l}27.03 \\
23.08\end{array}$ & & 9 & $\begin{array}{ll}3 & 12 \\
3 & \end{array}$ & $\begin{array}{l}21.04 \\
15.75\end{array}$ & $\begin{array}{r}21.13 \\
16.55\end{array}$ & 4 \\
\hline & $\begin{array}{ll}1 & 10 \\
1 & 10\end{array}$ & $\begin{array}{c}02.74 \\
08.59\end{array}$ & 31.04 & $\begin{array}{l}l_{14} \\
15\end{array}$ & $\begin{array}{l}3 \\
3\end{array}$ & $\begin{array}{l}11 \\
11\end{array}$ & $\begin{array}{l}\begin{array}{l}23.68 \\
34.9 C\end{array} \\
3.96\end{array}$ & $\begin{array}{l}24.72 \\
34.78\end{array}$ & $\begin{array}{l}11 \\
16\end{array}$ & $\begin{array}{ll}3 & 12 \\
3 & 12\end{array}$ & $\begin{array}{l}\begin{array}{l}15.75 \\
16.37\end{array}\end{array}$ & $\begin{array}{l}26.55 \\
17.63\end{array}$ & $\begin{array}{l}5 \\
1\end{array}$ \\
\hline & $\begin{array}{ll}1 & 10 \\
1 & 10\end{array}$ & $\begin{array}{l}0.039 \\
0\end{array}$ & 14.84 & 16 & 3 & 11 & 11.58 & 11.66 & 17 & $\begin{array}{ll}3 & 12 \\
3 & 12\end{array}$ & 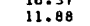 & 13.03 & $\frac{1}{2}$ \\
\hline & i 10 & $\quad 22.38$ & 20.02 & 17 & 3 & it & 12.25 & 11.59 & 20 & $\begin{array}{ll}3 & 12\end{array}$ & 11.98 & 10.41 & 3 \\
\hline & 110 & 37.81 & 36.06 & 19 & 3 & 11 & 16.31 & 15.18 & 21 & 312 & 13.86 & 11.55 & 4 \\
\hline & $\begin{array}{l}110 \\
110\end{array}$ & $\begin{array}{r}20.57 \\
0\end{array}$ & $\begin{array}{l}19.81 \\
13.31\end{array}$ & 21 & 3 & 11 & $\begin{array}{l}16.41 \\
\end{array}$ & $\begin{array}{l}16.02 \\
109.34\end{array}$ & 0 & 412 & $\begin{array}{l}14.48 \\
20.46\end{array}$ & $\begin{array}{l}15.52 \\
10.51\end{array}$ & 5 \\
\hline 0 & $\begin{array}{ll}1 & 10 \\
2 & 10\end{array}$ & $\begin{array}{l}0 \\
37.04\end{array}$ & - $\begin{array}{l}13.31 \\
40.65\end{array}$ & $\begin{array}{l}22 \\
23\end{array}$ & $\begin{array}{l}3 \\
3\end{array}$ & $\begin{array}{l}11 \\
11\end{array}$ & $\begin{array}{l}18.48 \\
13.09\end{array}$ & $\begin{array}{l}19.34 \\
14.05\end{array}$ & ${ }_{3}^{2}$ & $\begin{array}{ll}4 & 12 \\
4 & 12\end{array}$ & $\begin{array}{l}20 . c 6 \\
26.19\end{array}$ & $\begin{array}{l}19.51 \\
23.95\end{array}$ & $\begin{array}{l}6 \\
8\end{array}$ \\
\hline 1 & 210 & 29.54 & 29.36 & 1 & 4 & ii & 44.64 & 45.91 & 4 & 412 & 35.18 & 35.91 & 1 \\
\hline & $\begin{array}{ll}2 & 10 \\
2 & 10\end{array}$ & $\begin{array}{r}22.18 \\
4.89\end{array}$ & $\begin{array}{l}20.64 \\
42.65\end{array}$ & $\begin{array}{l}3 \\
5\end{array}$ & 4 & 4 & $\begin{array}{l}19.33 \\
42.66\end{array}$ & $\begin{array}{l}17.55 \\
44.38\end{array}$ & 5 & $\begin{array}{l}4 \\
4 \\
412\end{array}$ & $\begin{array}{l}\begin{array}{l}13.66 \\
22.01\end{array} \\
22\end{array}$ & $\begin{array}{l}12.97 \\
24.34\end{array}$ & 3 \\
\hline & $\begin{array}{ll}2 & 10 \\
2 & 10\end{array}$ & $\begin{array}{l}0.85 \\
61.17\end{array}$ & $\begin{array}{l}92.65 \\
61.87\end{array}$ & $\begin{array}{l}3 \\
6\end{array}$ & 4 & 11 & 28.81 & $\begin{array}{l}4.38 \\
28.02\end{array}$ & 7 & $4 \quad 12$ & $\begin{array}{l}22.61 \\
36.29\end{array}$ & 36.37 & 6 \\
\hline & 210 & 31.63 & $\begin{array}{l}1.87 \\
30.38\end{array}$ & $i$ & 4 & ii & 38.56 & 38.88 & 13 & 412 & $\begin{array}{l}30.49 \\
17.31\end{array}$ & 18.16 & ; \\
\hline & 210 & 59.75 & 59.02 & 8 & 4 & ii & 27.63 & 28.12 & 14 & 4 i2 & 19.94 & 21.49 & 0 \\
\hline 8 & $=10$ & 35.53 & 34.30 & 10 & 4 & it & $24.5 t$ & 25.62 & 19 & 412 & 14.51 & 14.13 & 1 \\
\hline 10 & $\begin{array}{l}210 \\
2\end{array}$ & $\begin{array}{l}51.84 \\
4\end{array}$ & 50.48 & ${ }_{12}^{11}$ & 4 & 11 & $\begin{array}{r}36.56 \\
15.40\end{array}$ & $\begin{array}{l}37.40 \\
14.14\end{array}$ & 21 & $\begin{array}{l}4 \quad 12 \\
5\end{array}$ & $\begin{array}{l}12.33 \\
22.52\end{array}$ & $\begin{array}{r}12.63 \\
23.98\end{array}$ & 2 \\
\hline & $\begin{array}{ll}2 & 10 \\
2 & 10\end{array}$ & $\begin{array}{c}46.39 \\
56.17\end{array}$ & $\begin{array}{l}\begin{array}{l}2.69 \\
52.79\end{array}\end{array}$ & $\begin{array}{l}12 \\
16\end{array}$ & 4 & ${ }_{11}^{n}$ & $\begin{array}{l}15.440 \\
21.79\end{array}$ & $\begin{array}{l}14.14 \\
22.70\end{array}$ & $\frac{1}{2}$ & $\begin{array}{ll}3 & 12 \\
5 & 12\end{array}$ & 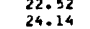 & $\begin{array}{l}23.98 \\
23.53\end{array}$ & 3 \\
\hline & $\begin{array}{ll}2 & 10 \\
2 & 10\end{array}$ & $\begin{array}{l}56.17 \\
31.60\end{array}$ & $\begin{array}{l}52.79 \\
33.15\end{array}$ & 17 & 4 & it & 27.55 & 27.99 & 3 & 512 & 11.10 & 14.24 & 9 \\
\hline & 210 & 33.92 & $\begin{array}{l}31.02 \\
31.02\end{array}$ & 18 & 4 & ii & 19.73 & 18.77 & 4 & $5 \quad 12$ & $18.8 \mathrm{C}$ & 19.85 & 1 \\
\hline 15 & 210 & $37 . \mathrm{cL}^{2}$ & 37.73 & 0 & 5 & ii & 35.54 & 35.64 & 6 & 512 & 8.50 & 10.13 & 5 \\
\hline & $\begin{array}{l}210 \\
2\end{array}$ & $\begin{array}{l}28.36 \\
20.75\end{array}$ & 28.82 & $\frac{1}{3}$ & $\begin{array}{l}5 \\
5\end{array}$ & 41 & 13.89 & $\begin{array}{l}22.31 \\
19.85\end{array}$ & 8 & $\begin{array}{l}512 \\
5\end{array}$ & $\begin{array}{l}20.88 \\
21.39\end{array}$ & $\begin{array}{r}20.45 \\
21.15\end{array}$ & \\
\hline & $\begin{array}{ll}2 & 10 \\
3 & 10\end{array}$ & $\begin{array}{l}2.75 \\
38.61\end{array}$ & $\begin{array}{l}\begin{array}{l}21.41 \\
38.41\end{array} \\
38.45\end{array}$ & ${ }_{4}^{3}$ & $\begin{array}{l}5 \\
5\end{array}$ & $\begin{array}{l}11 \\
11\end{array}$ & $\begin{array}{l}19.72 \\
25.49\end{array}$ & $\begin{array}{l}19.85 \\
24.83\end{array}$ & $\begin{array}{r}16 \\
5\end{array}$ & $\begin{array}{ll}5 & 12 \\
0 & 12\end{array}$ & $\begin{array}{l}21.39 \\
15.35\end{array}$ & $\begin{array}{l}21.15 \\
15.06\end{array}$ & \\
\hline 2 & $\begin{array}{ll}3 & 10\end{array}$ & $\begin{array}{l}30.01 \\
29.09\end{array}$ & 30.45 & 5 & 5 & 11 & 21.57 & 21.00 & $?$ & $\begin{array}{ll}0 \\
6 & 12\end{array}$ & 19.21 & 20.82 & \\
\hline & 10 & 29.24 & 30.88 & 6 & 5 & 11 & 40.02 & 41.62 & 13 & $6 \quad 12$ & 12.26 & 11.73 & \\
\hline & $\begin{array}{l}10 \\
10\end{array}$ & $\begin{array}{l}13.96 \\
25.09\end{array}$ & $13.8 \mathrm{t}$ & 7 & 5 & 11 & 15.88 & 15.61 & 14. & 612 & 10.53 & 11.28 & \\
\hline 6 & $\begin{array}{ll}3 & 10 \\
3 & 10\end{array}$ & 16.20 & $\begin{array}{l}15.14 \\
15.14\end{array}$ & $\begin{array}{l}8 \\
10\end{array}$ & 5 & i1 & $\begin{array}{l}10.15 \\
14.66\end{array}$ & 15.05 & 13 & 712 & 15.37 & 16.12 & \\
\hline 8 & 310 & $\quad 58.98$ & 57.60 & 11 & 5 & 11 & 10.67 & 9.91 & 4 & $\begin{array}{ll}0 & 13 \\
0 & 13\end{array}$ & 44.40 & 43.47 & \\
\hline 9 & $\begin{array}{l}3 \\
3\end{array}$ & $\begin{array}{r}29.19 \\
35.98\end{array}$ & 27.40 & 12 & $\begin{array}{c}5 \\
5\end{array}$ & 11 & 26.66 & $\begin{array}{l}26.70 \\
1 . t 4\end{array}$ & 6 & $\begin{array}{ll}0 & 13 \\
0 & 13\end{array}$ & $\begin{array}{l}11.47 \\
14.94\end{array}$ & $\begin{array}{r}9.16 \\
\end{array}$ & \\
\hline & $\begin{array}{ll}3 & 10 \\
3 & 10\end{array}$ & $\begin{array}{l}35.98 \\
28.46\end{array}$ & $\begin{array}{l}\begin{array}{l}35.08 \\
26.71\end{array} \\
26.08\end{array}$ & $\begin{array}{l}14 \\
19\end{array}$ & $\begin{array}{l}5 \\
5\end{array}$ & 11 & $\begin{array}{l}11.13 \\
11.61\end{array}$ & $\begin{array}{l}11.64 \\
13.59\end{array}$ & $\begin{array}{l}10 \\
12\end{array}$ & $\begin{array}{ll}0 & 13 \\
0 & 13\end{array}$ & & $\begin{array}{l}14.06 \\
44.25\end{array}$ & \\
\hline 37 & $\begin{array}{ll}3 & 10 \\
3 & 10\end{array}$ & $\begin{array}{l}8.40 \\
15.64\end{array}$ & $\begin{array}{l}28.11 \\
18.32\end{array}$ & $\begin{array}{r}19 \\
1\end{array}$ & $\begin{array}{l}3 \\
6\end{array}$ & $\begin{array}{l}11 \\
11\end{array}$ & $\begin{array}{l}11.01 \\
17 . t \in t\end{array}$ & 17.66 & 14 & $\begin{array}{lll}0 & 13 \\
0 & 13\end{array}$ & $\begin{array}{l}42.44 \\
42.64\end{array}$ & $\begin{array}{l}4.29 \\
40.07\end{array}$ & \\
\hline & 410 & 28.41 & $\begin{array}{r}29.73 \\
27.21\end{array}$ & 2 & 6 & 11 & 25.34 & $\begin{array}{r}24.94 \\
1.91\end{array}$ & 16 & $0 \quad 13$ & 41.55 & 39.72 & \\
\hline & $\begin{array}{l}4 \\
4 \\
10\end{array}$ & $\begin{array}{l}26.58 \\
46.7 €\end{array}$ & $\begin{array}{l}27.21 \\
48.93\end{array}$ & $\begin{array}{l}3 \\
4\end{array}$ & $\begin{array}{l}6 \\
6\end{array}$ & ${ }_{11}^{11}$ & $\begin{array}{l}11.55 \\
25.45\end{array}$ & $\begin{array}{l}110.91 \\
23.77\end{array}$ & $\begin{array}{l}22 \\
24\end{array}$ & $0 \quad 13$ & 19.79 & 20.33 & \\
\hline & 410 & 45.58 & 43.53 & 5 & 6 & 11 & 10.17 & 11.36 & $\begin{array}{r}24 \\
0\end{array}$ & $\begin{array}{ll}6 & 13\end{array}$ & 21,06 & 20.21 & \\
\hline 5 & 410 & 31.41 & 29.11 & 6 & 6 & i1 & 10.35 & 11.84 & i & $\begin{array}{lll}1 & 13\end{array}$ & 28.75 & 28.01 & \\
\hline 12 & 10 & 14.15 & 15.86 & $?$ & 6 & 11 & 18.45 & 17.11 & 2 & 123 & 16.85 & 14.62 & \\
\hline $\begin{array}{l}13 \\
15\end{array}$ & $\begin{array}{l}10 \\
10\end{array}$ & $\begin{array}{l}2.44 \\
34.79\end{array}$ & $\begin{array}{r}42.52 \\
34.49\end{array}$ & 14 & 6 & ${ }_{11}^{11}$ & $\begin{array}{l}14.41 \\
12 . \leqslant 3\end{array}$ & $\begin{array}{l}14.13 \\
14.83\end{array}$ & $\begin{array}{l}3 \\
6\end{array}$ & 13 & 27.61 & $\begin{array}{r}29.09 \\
231602\end{array}$ & \\
\hline & $\begin{array}{l}10 \\
10\end{array}$ & $\begin{array}{l}34.19 \\
15.00\end{array}$ & $\begin{array}{l}\begin{array}{l}3 t .49 \\
14.72\end{array} \\
142\end{array}$ & 27 & $\begin{array}{l}0 \\
6\end{array}$ & ${ }_{11}^{11}$ & 14.38 & $\begin{array}{l}14.03 \\
15.24\end{array}$ & $i$ & $\begin{array}{ll}1 & 13 \\
1 & 13\end{array}$ & $\begin{array}{l}\begin{array}{l}29.75 \\
30.30\end{array} \\
30\end{array}$ & $\begin{array}{l}26.02 \\
26.90\end{array}$ & \\
\hline & 10 & $\begin{array}{l}15.00 \\
17.63\end{array}$ & $\begin{array}{l}14.72 \\
19.64\end{array}$ & 0 & 7 & 11 & $18 . \varepsilon 3$ & 18.71 & 9 & 113 & $\begin{array}{l}37.17 \\
27.17\end{array}$ & 27.01 & \\
\hline & 10 & 27.10 & 28.25 & 2 & 7 & ii & 8.58 & 9.12 & 11 & $\begin{array}{ll}1 & 13\end{array}$ & 28.11 & 26.27 & \\
\hline & 10 & 27.61 & 28.66 & 4 & 7 & 11 & 11.35 & $\begin{array}{l}10.66 \\
1324\end{array}$ & 12 & 113 & 20.15 & 19.02 & \\
\hline & $\begin{array}{l}10 \\
10 \\
10\end{array}$ & $\begin{array}{l}13.13 \\
14.30\end{array}$ & $\begin{array}{l}16.14 \\
15.07\end{array}$ & 7 & 7 & 11 & 10.38 & 12.24 & 14 & 13 & $\begin{array}{l}12.19 \\
22.01\end{array}$ & $\begin{array}{l}11.5 \\
22.9\end{array}$ & \\
\hline & $\begin{array}{l}10 \\
10\end{array}$ & $\begin{array}{l}14.30 \\
36.77\end{array}$ & $\begin{array}{l}15.07 \\
36.0 B\end{array}$ & $\stackrel{8}{9}$ & 7 & $\begin{array}{l}11 \\
11\end{array}$ & $\begin{array}{l}7.5 \epsilon \\
9.87\end{array}$ & $\begin{array}{r}6.54 \\
11.29\end{array}$ & $\begin{array}{l}15 \\
16\end{array}$ & $\begin{array}{ll}1 & 13 \\
1 & 23\end{array}$ & $\begin{array}{l}\begin{array}{l}22.01 \\
19.62\end{array}\end{array}$ & $\begin{array}{l}22.12 \\
19.05\end{array}$ & \\
\hline & 10 & $\begin{array}{l}30.21 \\
20.21\end{array}$ & $\begin{array}{l}31.98 \\
21.93\end{array}$ & & & 12 & 24.73 & 24.87 & $\begin{array}{l}170 \\
17\end{array}$ & $\begin{array}{ll}1 & 13 \\
13\end{array}$ & 22.16 & 21.31 & \\
\hline & io & 19.75 & 21.27 & 6 & 0 & 12 & 36.51 & $\begin{array}{l}24.81 \\
36.45\end{array}$ & is & 13 & 15.85 & 15.28 & \\
\hline & 10 & 13.07 & 15.86 & 8 & 0 & 12 & 12.92 & 12.82 & 19 & 13 & 27.83 & 26.89 & \\
\hline & 10 & 19.29 & 18.52 & 10 & 0 & 12 & 72.82 & 72.02 & 21 & 13 & $\begin{array}{l}19.70 \\
8.90\end{array}$ & $\begin{array}{l}20.52 \\
9.26\end{array}$ & \\
\hline & $\begin{array}{l}10 \\
10\end{array}$ & $\begin{array}{l}\begin{array}{l}16.23 \\
16.34\end{array}\end{array}$ & $\begin{array}{l}17.01 \\
17.13\end{array}$ & 12 & 0 & 12 & $\begin{array}{l}87.69 \\
41.67\end{array}$ & $\begin{array}{l}87.31 \\
38.65\end{array}$ & 23 & 13 & $\begin{array}{r}8.90 \\
23.50\end{array}$ & $\begin{array}{r}9.26 \\
-234.46\end{array}$ & \\
\hline & $\begin{array}{l}10 \\
10\end{array}$ & 25.15 & 27.07 & $\begin{array}{l}16 \\
16\end{array}$ & $\begin{array}{l}0 \\
0\end{array}$ & 12 & $\begin{array}{l}41.67 \\
25.45\end{array}$ & $\begin{array}{l}38.65 \\
23.76\end{array}$ & $\frac{1}{2}$ & $\begin{array}{ll}2 & 13 \\
2 & 13\end{array}$ & $\begin{array}{l}\begin{array}{r}23.50 \\
33.59\end{array}\end{array}$ & $\begin{array}{l}24.46 \\
34.94\end{array}$ & \\
\hline & 10 & 16.05 & 16.31 & 18 & 0 & $\begin{array}{l}12 \\
12\end{array}$ & $\begin{array}{l}23.45 \\
24.79\end{array}$ & 23.13 & ${ }_{3}^{2}$ & 13 & $\begin{array}{l}19.73 \\
19.7 ?\end{array}$ & 18.36 & \\
\hline & 10 & 10.52 & 12.60 & 20 & 0 & 12 & 15.86 & 14.53 & 4 & $\begin{array}{ll}2 & 13\end{array}$ & 49.89 & 47.69 & \\
\hline & 10 & 15.49 & 16.19 & 22 & 0 & 12 & 19. & 18.31 & 5 & 213 & 34.44 & 31.68 & \\
\hline & $\begin{array}{l}11 \\
11\end{array}$ & $\begin{array}{l}\begin{array}{l}41.39 \\
33.28\end{array} \\
33.28\end{array}$ & $\begin{array}{l}2.55 \\
30.89\end{array}$ & 24 & i & 12 & $\begin{array}{l}14.55 \\
332\end{array}$ & $\begin{array}{l}14.02 \\
34.66\end{array}$ & 6 & $\begin{array}{ll}2 & 13 \\
2 & 13\end{array}$ & $\begin{array}{l}18.04 \\
10.18\end{array}$ & $\begin{array}{r}17.17 \\
15.68\end{array}$ & \\
\hline & $\begin{array}{ll}0 & 11 \\
0 & 11\end{array}$ & $\begin{array}{l}35.48 \\
75.43\end{array}$ & $\begin{array}{l}30.89 \\
77.59\end{array}$ & $\frac{1}{2}$ & & $\begin{array}{l}12 \\
12\end{array}$ & $\begin{array}{l}32.16 \\
28.33\end{array}$ & $\begin{array}{l}39.66 \\
29.45\end{array}$ & 17 & $\begin{array}{ll}2 & 13 \\
2 & 13\end{array}$ & $\begin{array}{l}16.18 \\
23.94 \\
23\end{array}$ & $\begin{array}{l}133.08 \\
24.09\end{array}$ & \\
\hline 12 & 11 & 9.92 & 8.45 & 3 & 1 & 12 & 29.58 & 30. & $\begin{array}{l}18 \\
18\end{array}$ & 213 & 14.24 & 14.94 & \\
\hline & $\begin{array}{l}11 \\
11 \\
11\end{array}$ & $\begin{array}{l}\begin{array}{l}20.15 \\
35.71\end{array} \\
3.75\end{array}$ & $\begin{array}{l}18.75 \\
.34 .82\end{array}$ & 4 & 1 & 12 & $\begin{array}{l}27.26 \\
49.18\end{array}$ & $\begin{array}{r}27.65 \\
\end{array}$ & 0 & $3 \quad 13$ & 46.10 & $\begin{array}{l}48.74 \\
16.78\end{array}$ & \\
\hline & $\begin{array}{ll}0 & 11 \\
0 & 11\end{array}$ & $\begin{array}{l}39.11 \\
49.32\end{array}$ & $\begin{array}{l}34.82 \\
49.00\end{array}$ & 8 & $\begin{array}{l}1 \\
1\end{array}$ & $\begin{array}{l}12 \\
12\end{array}$ & $\begin{array}{l}49.18 \\
20.43\end{array}$ & $\begin{array}{l}41.38 \\
19.20\end{array}$ & $\frac{1}{2}$ & $\begin{array}{cc}3 & 13 \\
3 & 13\end{array}$ & $\begin{array}{l}18.67 \\
32.22\end{array}$ & $\begin{array}{l}16.98 \\
32.99\end{array}$ & \\
\hline & 11 & 13.85 & 14.07 & 9 & $i$ & 12 & 35.55 & 34.25 & 3 & 313 & 21.32 & 20.55 & \\
\hline & 11 & 21.18 & 1 & 10 & i & 12 & 25.23 & 24. & 4 & $\begin{array}{ll}3 & 13 \\
3 & 3\end{array}$ & 13.29 & 14 & \\
\hline & $\begin{array}{l}11 \\
11\end{array}$ & $\begin{array}{l}14.22 \\
16.85\end{array}$ & $\begin{array}{l}15.23 \\
14.99\end{array}$ & 11 & 1 & 12 & $\begin{array}{l}52.79 \\
23.55\end{array}$ & 51 & 7 & $3 \quad 13$ & 29.64 & $\begin{array}{l}27.10 \\
14.05\end{array}$ & \\
\hline & 䇥 & $\begin{array}{l}\frac{16.85}{24.70} \\
24.70\end{array}$ & $\begin{array}{l}16.90 \\
24.74\end{array}$ & $\begin{array}{l}12 \\
14\end{array}$ & $\begin{array}{l}1 \\
1\end{array}$ & $\begin{array}{l}12 \\
12\end{array}$ & $\begin{array}{l}\begin{array}{r}23.55 \\
38.94\end{array} \\
38.9\end{array}$ & $\begin{array}{l}23.81 \\
37.27\end{array}$ & 19 & $\begin{array}{ll}3 & 13 \\
3 & \end{array}$ & 20.23 & $\begin{array}{l}14.05 \\
20.60\end{array}$ & \\
\hline & ii & $\begin{array}{l}12.02 \\
12.02\end{array}$ & $\begin{array}{l}24.14 \\
12.08\end{array}$ & $\begin{array}{l}14 \\
15\end{array}$ & 1 & $\begin{array}{l}12 \\
12\end{array}$ & $\begin{array}{l}38.94 \\
18.14\end{array}$ & 14.36 & $\begin{array}{l}19 \\
20\end{array}$ & $\begin{array}{ll}3 & 13 \\
3 & 13\end{array}$ & $\begin{array}{l}20.03 \\
19.02\end{array}$ & 29.85 & \\
\hline & ii & 10.40 & 8.57 & 16 & 1 & 12 & $10 . \varepsilon 5$ & 10.89 & 21 & $\begin{array}{ll}3 & 13\end{array}$ & 10.12 & 11.25 & \\
\hline & ii & 36.22 & 31.16 & 17 & 1 & 12 & 38. & 35.25 & 1 & 413 & 14.84 & 17 & \\
\hline & 11 & $\begin{array}{l}45.26 \\
13.68\end{array}$ & $\begin{array}{l}42.71 \\
14.99\end{array}$ & 18 & 1 & 12 & 10.26 & 12. & 3 & $4 \quad 13$ & 21 & $\begin{array}{l}20.23 \\
39.67\end{array}$ & \\
\hline & $\begin{array}{l}11 \\
11\end{array}$ & $\begin{array}{l}13.68 \\
23.17\end{array}$ & 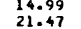 & $\begin{array}{l}19 \\
23\end{array}$ & $\frac{1}{1}$ & $\begin{array}{l}12 \\
12\end{array}$ & $\begin{array}{l}21.40 \\
19.72\end{array}$ & $\begin{array}{l}18.99 \\
18.64\end{array}$ & 5 & $4 \quad 13$ & $\begin{array}{l}39.55 \\
38.67\end{array}$ & $\begin{array}{l}35.67 \\
38.05\end{array}$ & \\
\hline & 11 & $\begin{array}{l}23.17 \\
19.00\end{array}$ & $\begin{array}{l}21.41 \\
19.41\end{array}$ & 25 & & 12 & 12.31 & $\begin{array}{l}18.64 \\
13.10\end{array}$ & $\begin{array}{l}3 \\
6 \\
6\end{array}$ & $\begin{array}{l}3 \\
3 \\
3\end{array}$ & $\begin{array}{l}38.67 \\
11.43\end{array}$ & $\begin{array}{l}38.74 \\
12.74\end{array}$ & \\
\hline & 11 & 9.20 & 9.22 & 0 & & 12 & 28.51 & 28.94 & 7 & $\begin{array}{ll}4 & 13\end{array}$ & 17.89 & 17.70 & \\
\hline & 12 & 5.89 & 8.91 & 1 & & 12 & 28.86 & 29.73 & 13 & 413 & 9.77 & 12.08 & \\
\hline$\frac{1}{2}$ & 11 & 32.23 & $\begin{array}{l}31.52 \\
22.331\end{array}$ & 2 & & 12. & $\leq 1.15$ & 52. & 15 & 13 & 14.04 & $\begin{array}{l}12.94 \\
26.02\end{array}$ & \\
\hline & $\begin{array}{l}11 \\
11\end{array}$ & $\begin{array}{l}21.16 \\
17.05\end{array}$ & $\begin{array}{l}22.31 \\
15.08\end{array}$ & 3 & & 12 & $\begin{array}{l}43.49 \\
28.43\end{array}$ & 46.32 & 0 & $\begin{array}{l}5 \\
5 \\
5\end{array}$ & $\begin{array}{r}28.16 \\
8.41\end{array}$ & $\begin{array}{r}26.02 \\
7.85\end{array}$ & \\
\hline & $\begin{array}{l}11 \\
11\end{array}$ & 10.41 & $\begin{array}{l}11.90 \\
11.90\end{array}$ & 6 & & ${ }_{12}^{12}$ & 10.47 & 10.68 & & & $\begin{array}{l}18.41 \\
18.91\end{array}$ & 21.04 & \\
\hline & ii & 25.10 & 24.54 & & 2 & 12 & 33.05 & 32.80 & & 13 & 19.05 & 21.74 & \\
\hline & & 9.60 & 10.68 & & & & 13.91 & 14.68 & & 13 & 15.80 & 16.04 & \\
\hline
\end{tabular}


lengths in the two molecules in the asymmetric unit decreased very significantly from the isotropic refinement to the final anisotropic refinement. For example, after the isotropic refinement, 7 of the 26 corresponding bond lengths deviated more than $0.06 \AA$ from each other, while after the anisotropic refinement, only 3 , possibly physically relevant ( $c f$. the discussion below) differences greater than $0.06 \AA$ remained. Therefore, if accurate parameter values are considered more important than reliable estimated standard deviations, based upon a simplified physical model, the present procedure seems well justified. It may be concluded that, although the number of independent structure factors, 1556, is too small to yield statistically valid e.s.d.'s from anisotropic refinement, the resultant parameters are physically more reasonable, implying that the measured structure factors are satisfactory. Thus, the suitability of the correction function applied to the relative optical densities is confirmed.

\section{RESULTS AND DISCUSSION OF THE STRUCTURE}

In the following discussion, atoms belonging to different asymmetric units are labelled as follows:

Superscript
None
i
ii
iii
iv
v

$$
\begin{aligned}
& \multicolumn{1}{c}{\text { Coordinates }} \\
& x, y, z \\
& \frac{1}{2}-x, y-\frac{1}{2}, z-\frac{1}{2} \\
& \frac{1}{2}+x, 1 \frac{1}{2}-y, z \\
& \frac{1}{2}+x, \frac{1}{2}-y, z \\
& 1-x, 1-y, z-\frac{1}{2} \\
& \frac{1}{2}-x, \frac{1}{2}+y, z-\frac{1}{2}
\end{aligned}
$$

Superscript
vi
vii
viii
ix
x

\section{Coordinates}

$$
\begin{aligned}
& \bar{x}, 1-y, \frac{1}{2}+z \\
& \frac{1}{2}-x, \frac{1}{2}+y, \frac{1}{2}+z \\
& \bar{x}, 1-y, z-\frac{1}{2} \\
& \bar{x}-\frac{1}{2}, 1 \frac{1}{2}-y, z \\
& \bar{x}-\frac{1}{2}, \frac{1}{2}-y, z
\end{aligned}
$$

A view of one of the molecules, showing the atom numbering and the angular shape of the isoalloxazine ring system, is given in Fig. 1.

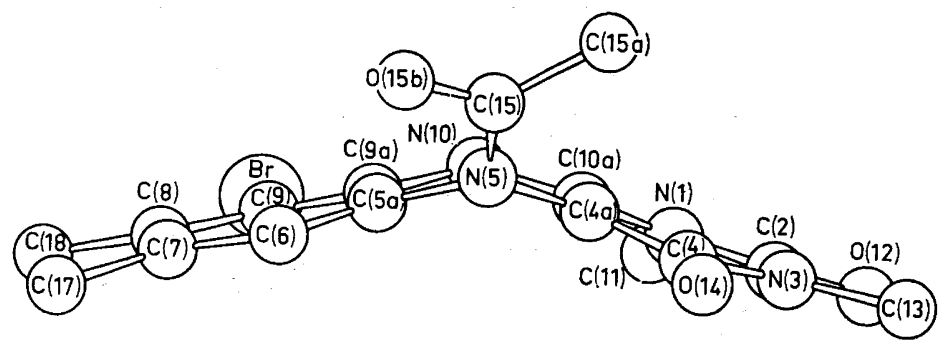

Fig. 1. Perspective view of 5-acetyl-1,3,7,8-tetramethyl-1,5-dihydroalloxazine.

The bond lengths are given in Table 6, and the angles are listed in Table 7. The assumed hydrogen positions are listed in Table 8 . Because of the refinement procedure used (see above), no attempts have been made to correct bond lengths for the effects of thermal motion. As can be seen from the r.m.s. components of the thermal displacements listed in Table 4, the deviations from isotropic temperature factors are not very pronounced. This may partly

Acta Chem. Scand. 25 (1971) No. 6 
Table 6. Bond lengths in $\AA$ and their calculated e.s.d.'s.

\begin{tabular}{|c|c|c|c|}
\hline & Mol. I & Mol. II & Average \\
\hline$N(1)-C(2)$ & $1.383(15)$ & $1.357 \quad(15)$ & $1.370(10)$ \\
\hline $\mathrm{N}(1)-\mathrm{C}(10 \mathrm{a})$ & 1.381 (13) & 1.362 (13) & $1.371 \quad(9)$ \\
\hline $\mathrm{N}(1)-\mathrm{C}(11)$ & 1.536 (18) & 1.468 (12) & $1.502(10)$ \\
\hline $\mathrm{C}(2)-\mathrm{N}(3)$ & $1.346(20)$ & 1.376 (13) & $1.361 \cdot(11)$ \\
\hline $\mathrm{C}(2)-\mathrm{O}(12)$ & $1.256(16)$ & 1.251 (14) & $1.253(10)$ \\
\hline $\mathrm{N}(3)-\mathrm{C}(4)$ & $1.412(15)$ & 1.421 (12) & $1.416 \quad(9)$ \\
\hline$N(3)-C(13)$ & 1.469 (16) & 1.449 (15) & $1.459(10)$ \\
\hline$C(4)-C(4 a)$ & 1.427 (15) & 1.394 (13) & $1.410 \quad(9)$ \\
\hline$C(4)-O(14)$ & $1.221 \quad(18)$ & 1.220 (11) & $1.220(10)$ \\
\hline$C(4 a)-N(5)$ & 1.398 (13) & 1.428 (12) & $1.413(8)$ \\
\hline$C(4 a)-C(10 a)$ & $1.354(17)$ & $1.383(12)$ & $1.368(10)$ \\
\hline$N(5)-C(5 a)$ & $1.455(11)$ & $1.433(13)$ & $1.444 \quad(8)$ \\
\hline$N(5)-C(15)$ & 1.379 (14) & 1.389 (12) & 1.384 \\
\hline$C(5 a)-C(6)$ & 1.419 (14) & 1.346 (15) & $1.382(10)$ \\
\hline$C(5 a)-C(9 a)$ & 1.347 (17) & 1.412 (13) & $1.379(10)$ \\
\hline$C(6)-C(7)$ & $1.372(14)$ & $1.392(16)$ & $1.382(10)$ \\
\hline$C(7)-C(8)$ & $1.399(18)$ & 1.359 (18) & 1.379 (12) \\
\hline$C(7)-C(17)$ & 1.521 (15) & 1.569 (19) & 1.545 (12) \\
\hline $\mathrm{C}(8)-\mathrm{C}(9)$ & $1.392(14)$ & $1.395(19)$ & $1.393(11)$ \\
\hline$C(8)-C(18)$ & 1.501 (15) & $1.532(21)$ & $1.516(12)$ \\
\hline $\mathrm{C}(9)-\mathrm{Br}$ & 1.929 (11) & $1.916(10)$ & 1.922 \\
\hline$C(9)-C(9 a)$ & $1.410(13)$ & $1.404(17)$ & $1.407(10)$ \\
\hline$C(9 a)-N(10)$ & 1.406 (12) & 1.389 (14) & $1.397 \quad(9)$ \\
\hline$N(10)-C(10 a)$ & $1.346(12)$ & 1.379 & 1.362 \\
\hline $\mathrm{C}(15)-\mathrm{C}(15 \mathrm{a})$ & 1.513 (14) & 1.467 (17) & 1.490 (11) \\
\hline$C(15)-O(15 b)$ & $1.227(13)$ & 1.232 (13) & $1.229 \quad(9)$ \\
\hline
\end{tabular}

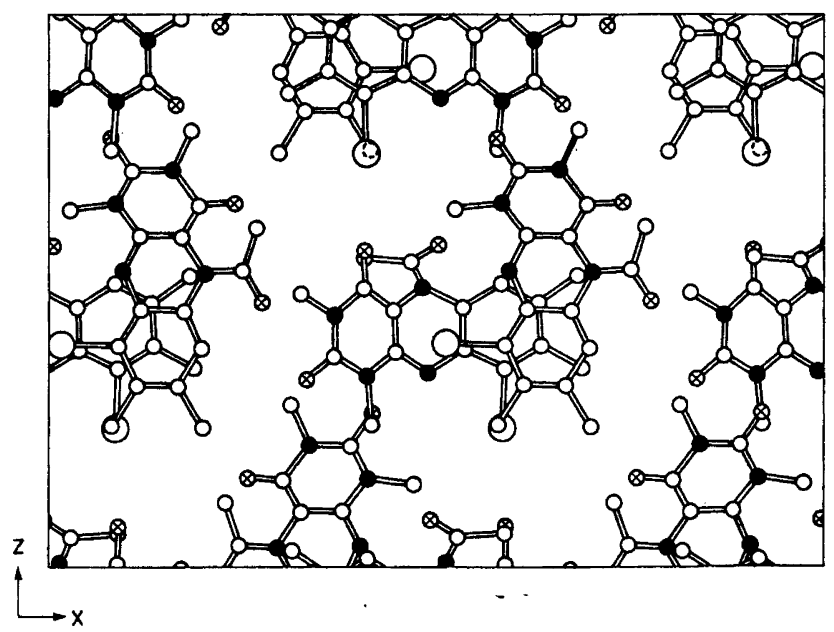

Fig. 2. Projection of the structure along the $b$ axis.

Acta Chem. Scand. 25 (1971) No. 6 
Table 7. Bond angles in degrees and their calculated e.s.d.'s.

\begin{tabular}{|c|c|c|c|}
\hline & Mol. I & Mol. II & Average \\
\hline$C(2)-N(1)-C(10 a)$ & $121.1(1.0)$ & $121.6(0.8)$ & $121.3(0.7)$ \\
\hline$C(2)-N(1)-C(11)$ & $117.9(1.0)$ & $118.9(1.0)$ & $118.4(0.7)$ \\
\hline$C(10 a)-N(1)-C(11)$ & $120.5(0.9)$ & $118.7(1.0)$ & $119.6(0.7)$ \\
\hline$N(1)-C(2)-N(3)$ & $118.0(1.1)$ & $117.5(1.0)$ & $117.7(0.7)$ \\
\hline $\mathrm{N}(1)-\mathrm{C}(2)-\mathrm{O}(12)$ & $120.2(1.3)$ & $122.0(1.0)$ & $121.1(0.8)$ \\
\hline $\mathrm{N}(3)-\mathrm{C}(2)-\mathrm{O}(12)$ & $121.8(1.1)$ & $120.5(1.1)$ & $121.1(0.8)$ \\
\hline $\mathrm{C}(2)-\mathrm{N}(3)-\mathrm{C}(4)$ & $124.2(1.0)$ & $123.9(0.9)$ & $124.0(0.7)$ \\
\hline $\mathrm{C}(2)-\mathrm{N}(3)-\mathrm{C}(13)$ & $119.5(1.0)$ & $119.0(0.9)$ & $119.3(0.7)$ \\
\hline $\mathrm{C}(4)-\mathrm{N}(3)-\mathrm{C}(13)$ & $116.3(1.1)$ & $117.1(0.8)$ & $116.7(0.7)$ \\
\hline$N(3)-C(4)-C(4 a)$ & $115.0(1.2)$ & $114.0(0.8)$ & $114.5(0.7)$ \\
\hline$N(3)-C(4)-O(14)$ & $121.5(1.0)$ & $119.1(0.9)$ & $120.3(0.7)$ \\
\hline$C(4 a)-C(4)-O(14)$ & $123.5(1.1)$ & $126.8(0.9)$ & $125.1(0.7)$ \\
\hline$C(4)-C(4 a)-C(10 a)$ & $121.0(1.1)$ & $121.7(0.8)$ & $121.4(0.7)$ \\
\hline $\mathrm{C}(4)-\mathrm{C}(4 \mathrm{a})-\mathrm{N}(5)$ & $120.8(1.0)$ & $121.4(0.9)$ & $121.1(0.7)$ \\
\hline$N(5)-C(4 a)-C(10 a)$ & $118.0(0.9)$ & $116.4(0.9)$ & $117.2(0.6)$ \\
\hline$C(4 a)-N(5)-C(5 a)$ & $112.5(0.9)$ & $117.2(0.8)$ & $114.8(0.6)$ \\
\hline$C(4 a)-N(5)-C(15)$ & $124.0(0.8)$ & $122.4(0.9)$ & $123.2(0.6)$ \\
\hline$C(5 a)-N(5)-C(15)$ & $118.0(0.8)$ & $118.7(0.9)$ & $118.4(0.6)$ \\
\hline$N^{\prime}(5)-C(5 a)-C(6)^{\prime}$ & $121.3(1.1)$ & $126.0(0.9)$ & $123.7(0.7)$ \\
\hline$N(5)-C(5 a)-C(9 a)$ & $117.7(0.9)$ & $114.3(0.9)$ & $116.0(0.6)$ \\
\hline$C(6)-C(5 a)-C(9 a)$ & $120.8(0.9)$ & $119.6(1.0)$ & $120.2(0.7)$ \\
\hline$C(5 a)-C(6)-C(7)^{\prime}$ & $119.5(1.1)$ & $121.5(1.0)$ & $120.5(0.8)$ \\
\hline$C(6)-C(7)-C(8)$ & $121.2(1.1)$ & $121.4(1.1)$ & $121.3(0.8)$ \\
\hline$C(6)-C(7)-C(17)$ & $117.9(1.1)$ & $116.7(1.1)$ & $117.3(0.8)$ \\
\hline$C(8)-C(7)-C(17)$ & $121.0(0.9)$ & $121.9(1.1)$ & $121.4(0.7)$ \\
\hline$C(7)-C(8)-C(9)$ & $117.3(0.9)$ & $117.5(1.1)$ & $117.4(0.7)$ \\
\hline$C(7)-C(8)-C(18)$ & $123.7(1.0)$ & $121.5(1.2)$ & $122.6(0.8)$ \\
\hline$C(9)-C(8)-C(18)$ & $119.0(1.1)$ & $121.0(1.2)$ & $120.0(0.8)$ \\
\hline$C(8)-C(9)-C(9 a)$ & $122.2(1.1)$ & $122.3(1.0)$ & $122.3(0.7)$ \\
\hline $\mathrm{C}(8)-\mathrm{C}(9)-\mathrm{Br}$ & $121.6(0.8)$ & $120.0(1.0)$ & $120.8(0.7)$ \\
\hline $\mathrm{C}(9 \mathrm{a})-\mathrm{C}(9)-\mathrm{Br}$ & $115.8(0.8)$ & $117.6(1.0)$ & $116.7(0.6)$ \\
\hline$C(5 a)-C(9 a)-C(9)$ & $118.5(0.9)$ & $117.5(1.0)$ & $118.0(0.7)$ \\
\hline$C(5 a)-C(9 a)-N(10)$ & $119.5(0.8)$ & $120.7(1.0)$ & $120.1(0.6)$ \\
\hline$C(9)-C(9 a)-N(10)$ & $122.0(1.0)$ & $121.8(0.9)$ & $121.9(0.7)$ \\
\hline$C(9 a)-N(10)-C(10 a)$ & $117.0(0.9)$ & $117.0(0.8)$ & $117.0(0.6)$ \\
\hline$N(1)-C(10 a)-C(4 a)$ & $120.3(0.9)$ & $120.3(0.9)$ & $120.3(0.7)$ \\
\hline$N(1)-C(10 a)-N(10)$ & $117.8(1.0)$ & $119.2(0.8)$ & $118.5(0.6)$ \\
\hline$C(4 a)-C(10 a)-N(10)$ & $121.8(0.9)$ & $120.3(0.9)$ & $121.0(0.7)$ \\
\hline$N(5)-C(15)-C(15 a)$ & $116.4(0.9)$ & $117.8(0.9)$ & $117.1(0.7)$ \\
\hline$N(15)-C(15)-O(15 b)$ & $121.7(0.9)$ & $120.6(1.0)$ & $121.2(0.7)$ \\
\hline$C(15 a)-C(15)-O(15 b)$ & $121.8(1.1)$ & $121.4(1.0)$ & $121.6(0.7)$ \\
\hline
\end{tabular}

be a result of the refinement procedure. On the other hand, it has been found that the thermal ellipsoids, as visualized by use of the plot program ORTEP, ${ }^{15}$ give a plausible pattern of ellipsoid axial directions.

The equations of least-squares planes through the pyrimidine and benzene rings in the two molecules are given in Table 9, together with the distances from the planes. The calculated angles between the planes are $28.8^{\circ}$ and $31.7^{\circ}$.

From Table 6 it may be concluded that the differences between the bond lengths in the two molecules cannot be explained only by considering statistical

Acta Chem. Scand. 25 (1971) No. 6 
Table 8. Fractional coordinates for hydrogen atoms.

\begin{tabular}{|c|c|c|c|c|}
\hline $\begin{array}{l}\text { Atom } \\
\text { Mol. I }\end{array}$ & Bonded to & $x / a$ & $y / a$ & $z / a$ \\
\hline $\begin{array}{l}\mathbf{H}(1) \\
H(2) \\
H(3) \\
H(4) \\
H(5) \\
H(6) \\
H(7) \\
H(8) \\
H(9) \\
H(10) \\
H(11) \\
H(12) \\
H(13) \\
H(14) \\
H(15) \\
H(16) \\
H(17)\end{array}$ & $\begin{array}{l}C(6) \\
N(10) \\
C(11) \\
C(11) \\
C(11) \\
C(13) \\
C(13) \\
C(13) \\
C(15 a) \\
C(15 a) \\
C(15 a) \\
C(17) \\
C(17) \\
C(17) \\
C(18) \\
C(18) \\
C(18)\end{array}$ & $\begin{array}{l}0.5760 \\
0.4870 \\
0.4113 \\
0.4454 \\
0.4078 \\
0.3230 \\
0.3351 \\
0.3187 \\
0.4090 \\
0.4028 \\
0.4152 \\
0.6880 \\
0.6860 \\
0.6910 \\
0.6780 \\
0.6868 \\
0.7073\end{array}$ & $\begin{array}{l}0.5000 \\
0.6300 \\
0.7803 \\
0.7095 \\
0.5880 \\
0.8600 \\
0.0497 \\
0.9189 \\
0.2453 \\
0.4486 \\
0.1970 \\
0.4600 \\
0.5100 \\
0.3260 \\
0.4261 \\
0.2981 \\
0.3889\end{array}$ & $\begin{array}{l}0.5680 \\
0.3360 \\
0.2864 \\
0.2425 \\
0.2514 \\
0.5400 \\
0.4895 \\
0.4588 \\
0.5030 \\
0.5739 \\
0.5777 \\
0.4950 \\
0.5590 \\
0.5300 \\
0.3082 \\
0.3417 \\
0.3866\end{array}$ \\
\hline \multicolumn{5}{|c|}{ Mol. II } \\
\hline $\begin{array}{l}H(1) \\
H(2) \\
H(3) \\
H(4) \\
H(5) \\
H(6) \\
H(7) \\
H(8) \\
H(9) \\
H(10) \\
H(11) \\
H(12) \\
H(13) \\
H(14) \\
H(15) \\
H(16) \\
H(17)\end{array}$ & $\begin{array}{l}C(6) \\
N(10) \\
C(11) \\
C(11) \\
C(11) \\
C(13) \\
C(13) \\
C(13) \\
C(15) \\
C(15) \\
C(15) \\
C(17) \\
C(17) \\
C(17) \\
C(18) \\
C(18) \\
C(18)\end{array}$ & $\begin{array}{l}0.2385 \\
0.0552 \\
0.0254 \\
0.0130 \\
0.0134 \\
0.2298 \\
0.1758 \\
0.1750 \\
0.2776 \\
0.2630 \\
0.2839 \\
0.2294 \\
0.1770 \\
0.2216 \\
0.1095 \\
0.0630 \\
0.0794\end{array}$ & $\begin{array}{l}0.6526 \\
0.4961 \\
0.7273 \\
0.5600 \\
0.6350 \\
0.8050 \\
0.7822 \\
0.9260 \\
0.3191 \\
0.4171 \\
0.5555 \\
0.5881 \\
0.6355 \\
0.7734 \\
0.5367 \\
0.6460 \\
0.4263\end{array}$ & $\begin{array}{l}0.3974 \\
0.5257 \\
0.6189 \\
0.6180 \\
0.6180 \\
0.7634 \\
0.8269 \\
0.7970 \\
0.6021 \\
0.6516 \\
0.6223 \\
0.2277 \\
0.2752 \\
0.2786 \\
0.2201 \\
0.2530 \\
0.2561\end{array}$ \\
\hline
\end{tabular}

errors, unless bigger standard deviations than those calculated from the least-squares refinement are used. For instance, a $\chi^{2}$-test of the kind proposed by Hamilton ${ }^{16}$ on all "equivalent" pairs of bond lengths shows that the standard deviations should be 2.05 times larger if the molecules should be considered identical at a significance level of 0.95 . This result may in part be due to the great number of parameters varied in the least-squares refinement. It may be noted, however, that for all three pairs of bonds, $N(1)-C(11)$, $\mathrm{C}(5 \mathrm{a})-\mathrm{C}(6)$, and $\mathrm{C}(5 \mathrm{a})-\mathrm{C}(9 \mathrm{a})$, which deviate more than $0.06 \AA$, differences between the molecules depending on different bromine interactions cannot be excluded. The packing of the molecules projected on (010) is shown in Fig. 2, 
Table 9. Least-squares planes. The planes are described, using a vector basis $(m, n, p)$, having $m\|a, n\| b$, and $p \| c$.

Plane A. Molecule I $\quad 0.5902 m+0.8071 n+0.0159 p=10.335$

Molecule II $-0.1936 m+0.8837 n-0.4261 p=-1.180$

Plane B. Molecule I $\quad 0.1295 m+0.9915 n-0.0100 p=5.304$

Molecule II $-0.2126 m+0.9705 n+0.1133 p=4.198$

The angle between plane $A$ and plane $B$ is $28.8^{\circ}$ in molecule I, and $31.7^{\circ}$ in molecule II.

\begin{tabular}{|c|c|c|c|c|c|}
\hline \multicolumn{3}{|c|}{ Plane A } & \multicolumn{3}{|c|}{ Plane B } \\
\hline \multirow{2}{*}{ Atom } & \multicolumn{2}{|c|}{ Deviation $^{a}$} & \multirow{2}{*}{ Atom } & \multicolumn{2}{|c|}{ Deviation $^{a}$} \\
\hline & Mol. I & Mol. II & & Mol. I & Mol. II \\
\hline$N(1)$ & -47 & 20 & $\mathrm{~N}(5)$ & -126 & -53 \\
\hline $\mathrm{C}(2)$ & -58 & -18 & $\mathrm{C}(5 \mathrm{a})$ & 27 & -1 \\
\hline $\mathbf{N}(3)$ & 35 & -35 & $\mathrm{C}(6)$ & 110 & 45 \\
\hline $\mathrm{C}(4)$ & 101 & 95 & $\mathrm{C}(7)$ & 16 & 24 \\
\hline$C(4 a)$ & -34 & -42 & $\mathrm{C}(8)$ & -78 & -42 \\
\hline$N(5)$ & -86 & -28 & $\mathrm{C}(9)$ & -36 & -19 \\
\hline$N(10)$ & 102 & 38 & $\mathrm{C}(9 \mathrm{a})$ & 7 & 3 \\
\hline$C(10 a)$ & -13 & -31 & $\mathrm{~N}(10)$ & $\begin{array}{r}79 \\
2 \cdot 0\end{array}$ & 43 \\
\hline & & & & -250 & -14 \\
\hline
\end{tabular}

a Deviations given in $10^{-3} \AA$.

$b$ The bromine atoms not included in the least-squares planes.

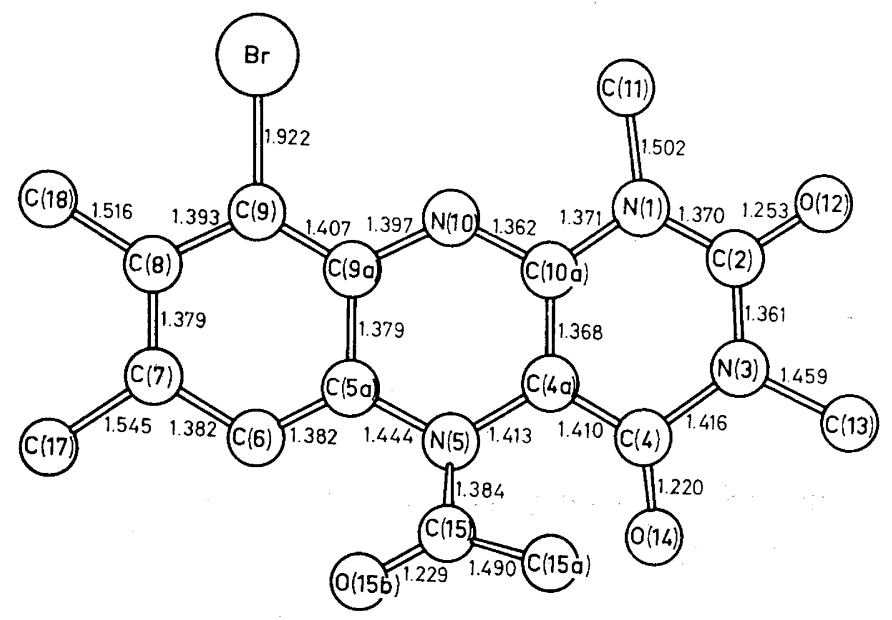

Fig. 3. Interatomic (average) distances (in $\AA$ ) in 5-acetyl-1,3,7,8-tetramethyl-1,5dihydroalloxazine.

and all intermolecular distances less than $3.80 \AA$ are given in Table 10. As can be seen from this figures, the bromine atoms have very different surroundings. Furthermore, two possible hydrogen bonds, $\mathrm{N}(10)(\mathrm{mol}$. I)...O(12)(i)(mol. II), Acta Chem. Scand. 25 (1971) No. 6 
Table 10. Intermolecular distances shorter than 3.8 $\AA$. The first figure after the chemical symbol is the molecule numbering.

\begin{tabular}{|c|c|c|c|c|c|c|c|c|c|c|}
\hline $\mathrm{Br} 1$ & ...O1 & (14) & (iv) & $3.37 \AA$ & $\mathrm{Cl}$ & (11) & ...C2 & (2) & (i) & 3.78 \\
\hline & $\ldots \mathrm{C} 2$ & (18) & (iii) & 3.69 & & & $\ldots \mathrm{O} 2$ & (i2) & & 3.39 \\
\hline \multirow{7}{*}{$\mathrm{Br} 2$} & ...N1 & (1) & (ix) & 3.74 & $\mathrm{O} 1$ & (12) & $\ldots \mathrm{C} 2$ & (2) & (v) & 3.67 \\
\hline & ...C1 & $(4 a)$ & (ix) & 3.77 & & & ...N2 & (3) & (v) & 3.61 \\
\hline & ...Cl & (5a) & $(\mathrm{x})$ & 3.76 & & & ...C2 & (13) & (v) & 3.67 \\
\hline & $\ldots \mathrm{Cl}$ & (9a) & (x) & 3.55 & $\mathrm{Cl}$ & (13) & $\ldots 02$ & (14) & & 3.73 \\
\hline & .... & $(10 a)$ & (ix) & 3.59 & & & ...C2 & (15) & & 3.77 \\
\hline & $\ldots 01$ & (15b) & $(\mathrm{x})$ & 3.68 & & & $\ldots 02$ & (15b) & & 3.67 \\
\hline & ...O2 & (12) & (viii) & 3.65 & Ol & (14) & ...C2 & (11) & (ii) & 3.41 \\
\hline \multirow{2}{*}{ N1 (1) } & ...O2 & (12) & (i) & 3.49 & $\mathrm{Cl}$ & $(15 a)$ & )...C2 & (15a) & & 3.72 \\
\hline & ...C2 & (13) & (i) & 3.78 & & & $\ldots 02$ & (15b) & & 3.71 \\
\hline N1 (3) & ...O2 & (15b) & & 3.56 & 01 & $(15 b)$ & )...N2 & (10) & (iii) & 3.15 \\
\hline \multirow[t]{3}{*}{$\mathrm{Cl}(6)$} & ...C2 & (9a) & (ii) & 3.77 & & & ...C2 & (11) & (iii) & 3.23 \\
\hline & ...N2 & (10) & (ii) & 3.72 & $\mathrm{Cl}$ & (17) & $\ldots \mathrm{C} 2$ & (4) & (ii) & 3.47 \\
\hline & ...C2 & $(10 a)$ & (ii) & 3.65 & & & $\ldots \mathrm{C} 2$ & (4a) & (ii) & 3.60 \\
\hline \multirow[t]{3}{*}{ Cl (7) } & $\ldots \mathrm{C} 2$ & $(5 a)$ & (ii) & 3.68 & & & ...N2 & (5) & (iii) & 3.77 \\
\hline & $\ldots \mathrm{C} 2$ & $(9 a)$ & (iii) & 3.69 & & & ...N2 & (5) & (ii) & 3.79 \\
\hline & ...N2 & (10) & (iii) & 3.71 & & & ...O2 & (14) & (ii) & 3.48 \\
\hline \multirow{4}{*}{ C1 (8) } & .... 2 & (6) & (ii) & 3.66 & & & ...C2 & (15) & (iii) & 3.51 \\
\hline & ...C2 & (7) & (ii) & 3.69 & & & ...C2 & (15a) & (iii) & 3.69 \\
\hline & $\ldots \mathrm{C} 2$ & (9) & (iii) & 3.69 & & & $\ldots 02$ & $(15 \mathrm{~b})$ & (iii) & 3.7 \\
\hline & ...C2 & (9a) & (iii) & 3.77 & $\mathrm{C} 1$ & (18) & $\ldots \mathrm{C} 2$ & (6) & (ii) & 3.69 \\
\hline \multirow[t]{3}{*}{$\mathrm{Cl}(9)$} & $\ldots \mathrm{C} 2$ & $(7)$ & (ii) & 3.78 & & & $\ldots \mathrm{C} 2$ & (7) & (ii) & 3.73 \\
\hline & ...C2 & (8) & (ii) & 3.64 & & & $\ldots \mathrm{C} 2$ & (13) & (iv) & 3.74 \\
\hline & $\ldots \mathrm{C} 2$ & (9) & (iii) & 3.77 & $\mathrm{C} 2$ & (11) & $\ldots \mathrm{C} 2$ & (18) & (vi) & 3.59 \\
\hline N1 (10) & ...O2 & (12) & (i) & 2.97 & $\mathrm{C} 2$ & (13) & $\ldots 02$ & (15b) & (vii) & 3.60 \\
\hline & )...O2 & (12) & (i) & 3.34 & $\mathrm{O} 2$ & (14) & $\ldots \mathrm{C} 2$ & (17) & (vii) & 3.56 \\
\hline \multirow[t]{2}{*}{ Cl (11) } & ...01 & (15b) & (iv) & 3.59 & C2 & (15a) & 4)...C2 & (17) & (vii) & 3.23 \\
\hline & ...N2 & (1) & (v) & 3.69 & & & & & & \\
\hline
\end{tabular}

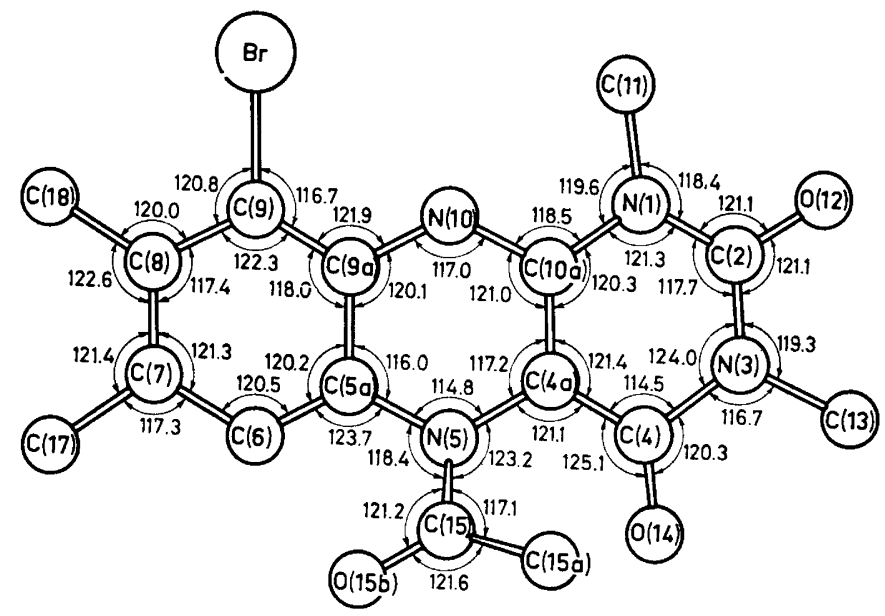

Fig. 4. (Average) bond angles in 5-acetyl-1,3,7,8-tetramethyl-1,5-dihydroalloxazine. 
$2.97 \AA$, and $\mathrm{N}(10)(\mathrm{iii})(\mathrm{mol} \mathrm{II}) \ldots \mathrm{O}(\mathrm{l} \mathrm{bb})(\mathrm{mol} \mathrm{I}), 3.15 \AA$, are found in the structure. It seems reasonable in any event to multiply the standard deviations by a factor of at least 1.5.

A comparison between the structure of 5-acetyl-9-bromo-1,3,7,8,10-pentamethyl-1,5-dihydro-isoalloxazine ${ }^{1}$ (hereafter called the penta-compound) and the present one shows that only one bond length, $\mathrm{C}(2)-\mathrm{N}(3)$, in the pentacompound, $1.43(3) \AA$, is possibly significantly different from the corresponding "average molecule" (cf. Tables $6-7$ and Figs. 3-4) bond distance, 1.361(11) $\AA$, in the present structure $\left(\Delta /\left(\sigma_{1}{ }^{2}+\sigma_{2}{ }^{2}\right)^{\sharp}=(1.430-1.361) /\left(0.03^{2}+(1.5 \times 0.011)^{2}\right)^{\sharp}=\right.$ $=2.01) .{ }^{17}$ On the other hand, in other reduced flavin structures, the $\mathrm{C}(2)-\mathrm{N}(3)$ bonds are in agreement with the present structure. In 9-bromo-1,3,7,8,10pentamethyl-1,5-dihydro-isoalloxazine ${ }^{18}$ and 5-diethyl-3,7,8,10-tetramethyl1,5-dihydro-isoalloxazine, ${ }^{5}$ the $\mathrm{C}(2)-\mathrm{N}(3)$ bond distances found are $1.375 \AA$ and $1.378 \AA$, respectively. 22 of the 26 corresponding bond lengths in the present "average molecule" ( $c f$. Table 6) and the penta-compound differ by less than one calculated standard deviation for the penta-compound, and of the remaining four bonds, three deviate less than two standard deviations, while the fourth is the $\mathrm{C}(2)-\mathrm{N}(3)$ bond discussed above.

As expected for reduced flavins, some double bond character is found in the $\mathrm{C}(4 \mathrm{a})-\mathrm{C}(10 \mathrm{a})$ bonds. The conclusion drawn by Werner et al., ${ }^{5}$ that in reduced flavins, $\pi$-electrons seem to be localized also in the bond $C(4)-C(4 a)$, is supported by the present structure determination.

The calculated angle between the least-squares planes through the pyrimidine and benzene rings in the penta-compound is $35.5^{\circ}$. Given the large e.s.d.'s in the latter compound, no significant change in the alloxazine ring system due to a methyl group at $\mathrm{N}(10)$ can be demonstrated. It also seems probable that the angular shape of the molecule ( $c f$. Fig. I) is determined entirely by the electron configuration at $\mathrm{N}(5)$.

The relevance of the structure of the reduced alloxazine ring system in biological redox systems is discussed in the publication of the penta-compound. ${ }^{1}$

Finally, it may be noted that the C(aromatic) - Br bond length, $1.922 \AA$, is more in agreement with the average $1.903 \AA$ obtained from 13 structure determinations, published in Acta Crystallographica January 1969 - July 1970, than with the value $1.85 \pm 0.01$, reported by Sutton. ${ }^{19}$ This may be somewhat dependent on other substituents on the benzene rings, but it seems notable that only one of the $\mathrm{C}-\mathrm{Br}$ bond lengths reported in the 13 structure determinations mentioned above is less than $1.85 \AA$.

Acknowledgements. The present investigation was financially supported by the TriCentennial Fund of Bank of Sweden, and by the Swedish Natural Science Research Council. The authors are indebted to Professor Peder Kierkegaard for his interest in this work. The authors are also indebted to Dr. Lajos Maròn for the supply of the crystals used, and to Dr. Donald F. Koenig for correcting the English of the manuscript.

\section{REFERENCES}

1. Werner, P. E. and Rönnquist, O. Acta Chem. Scand. 24 (1970) 997.

2. Abrahamsson, S. J. Sci. Instr. 43 (1966) 931.

3. Werner, P.-E. Arkiv Kemi 31 (1969) 505.

4. Werner, P.-E. Acta Cryst. A 26 (1970) 489.

Acta Chem. Scand. 25 (1971) No. 6 
5. Werner, P.-E., Linnros, B. and Leijonmarck, M. Acta Chem. Scand. 25 (1971) 1297

6. Mammi, M., Bardi, R. and Bezzi, S. Acta Cryst. 16 (1963) Al49.

7. Werner, P.-E. Arkiv Kemi 31 (1969) 513.

8. SAAB Film Scanner Manual, 1967.

9. Monahan, J. E., Schiffer, M. and Schiffer, J. P. Acta Cryst. 22 (1967) 322.

10. Morimoto, H. and Uyeda, R. Acta Cryst. 11 (1963) 1107.

11. IUCr World List of Crystallographic Computer Programs, 2nd Ed., Cambridge, Mass. 1966 , No. 384.

12. Freeman, A. J. Acta Cryst. 12 (1959) 261.

13. Cromer, D. T. and Waber, J. T. Acta Cryst. 18 (1965) 104.

14. Cruickshank, D. W. J., Pilling, D. E., Bujosa, A., Lovell, F. M. and Truter, M. R. Computing Methods and the Phase Problem in X-ray Crystal Structure Analysis, Paper 6, Pergamon, Oxford 1961.

15. Johnson, C. K. ORTEP, ORNL-3794, Oak Ridge National Laboratory, Oak Ridge, Tenn. 1965.

16. Hamilton, W. C. Acta Cryst. A 25 (1969) 194.

17. Cruickshank, D. W. J. and Robertson, A. P. Acta Cryst. 6 (1953) 698.

18. Norrestam, R. and von Glehn, M. Acta Cryst. In press.

19. Sutton, L. E. Special Publication No. 18, The Chemical Society, London 1965.

Received November 16, 1970. 